\title{
A NONEXISTENCE RESULT FOR YAMABE TYPE PROBLEMS ON THIN ANNULI
}

\section{UN RÉSULTAT DE NON-EXISTENCE POUR UN PROBLÈME DE TYPE YAMABE SUR DES ANNEAUX MINCES}

\section{Mohamed BEN AYED ${ }^{\text {a }}$, Khalil EL MEHDI ${ }^{\mathrm{b}}$, Mokhless HAMMAMI ${ }^{\mathrm{a}}$ \\ a Département de Mathématiques, Faculté des Sciences de Sfax, Route Soukra, Sfax, Tunisia ${ }^{\mathrm{b}}$ Centre de Mathématiques, Ecole Polytechnique, 91128 Palaiseau Cedex, France}

Received 5 November 2001

ABSTRACT. - Given any constant $C>0$, we show that there exists $\varepsilon_{0}>0$ such that for any $\varepsilon<\varepsilon_{0}$, the problem $P_{\varepsilon}:-\Delta u_{\varepsilon}=u_{\varepsilon}^{(n+2) /(n-2)}, u_{\varepsilon}>0$ in $A_{\varepsilon} ; u_{\varepsilon}=0$ on $\partial A_{\varepsilon}$, has no solution $u_{\varepsilon}$, whose energy, $\int_{A_{\varepsilon}}\left|\nabla u_{\varepsilon}\right|^{2}$, is less than C, where $A_{\varepsilon}$ is a ringshaped open set in $\mathbb{R}^{n}$ and $n \geqslant 4$. (C) 2002 L'Association Publications de l'Institut Henri Poincaré. Published by Elsevier B.V. All rights reserved

AMS classification: Primary 35J65, Secondary 58E05; 35B40

Keywords: Noncompact variational problems; Elliptic problems with critical Sobolev exponent; Asymptotic behavior of solutions

RÉSUMÉ. - Etant donné une constante positive $C$ arbitraire, nous montrons qu'il existe $\varepsilon_{0}>0$ tel que pour tout $\varepsilon<\varepsilon_{0}$, le problème $P_{\varepsilon}:-\Delta u_{\varepsilon}=u_{\varepsilon}^{(n+2) /(n-2)}, u_{\varepsilon}>0$ dans $A_{\varepsilon} ; u_{\varepsilon}=0$ sur $\partial A_{\varepsilon}$, ne possède pas de solution $u_{\varepsilon}$ dont l'energie, $\int_{A_{\varepsilon}}\left|\nabla u_{\varepsilon}\right|^{2}$, est plus petite que $\mathrm{C}$, où $A_{\varepsilon}$ est un ouvert de $\mathbb{R}^{n}$ ayant la forme d'un anneau et $n \geqslant 4$.

(c) 2002 L'Association Publications de l'Institut Henri Poincaré. Published by Elsevier B.V. All rights reserved

\section{Introduction and the main results}

Let us consider the nonlinear elliptic problem

$$
P(\Omega) \quad\left\{\begin{array}{lll}
-\Delta u=u^{p}, & u>0 & \text { in } \Omega, \\
u=0 & \text { on } \partial \Omega,
\end{array}\right.
$$

E-mail addresses: mohamed.benayed@fss.rnu.tn (M. Ben Ayed), elmehdi@math.polytechnique.fr (K. El Mehdi), mokhless.hammami@fss.rnu.tn (M. Hammami). 
where $\Omega$ is a bounded regular domain in $\mathbb{R}^{n}, n \geqslant 3$ and $p+1=2 n /(n-2)$ is the critical Sobolev exponent.

The interest in this type of equation comes from its resemblance to some nonlinear problems in geometry (Yamabe problem, Harmonic maps,...) and physics (YangMills equations, The $n$ body problem,...) where some lack of compactness occurs (see Brezis [6]). It is well known that if $\Omega$ is starshaped, $P(\Omega)$ has no solution (see Pohozaev [13]) and if $\Omega$ has nontrivial topology, in the sense that $H_{2 k-1}(\Omega ; Q) \neq 0$ or $H_{k}(\Omega ; Z / 2 Z) \neq 0$ for some $k \in \mathbb{N}$, Bahri and Coron [3] have shown that $P(\Omega)$ has a solution. Nevertheless, Ding [9] (see also Dancer [8]) gave the example of contractible domain on which $P(\Omega)$ has a solution. Then, the question related to existence or nonexistence of solution of $P(\Omega)$ remained open.

In this paper, we study the problem $P(\Omega)$ when $\Omega=A_{\varepsilon}$ is a ringshaped open set in $\mathbb{R}^{n}$ and $\varepsilon \rightarrow 0$. More precisely, let $f$ be any smooth function:

$$
f: \mathbb{R}^{n-1} \rightarrow[1,2], \quad\left(\theta_{1}, \ldots, \theta_{n-1}\right) \rightarrow f\left(\theta_{1}, \ldots, \theta_{n-1}\right)
$$

which is periodic of period $\pi$ with respect to $\theta_{1}, \ldots, \theta_{n-2}$ and of period $2 \pi$ with respect to $\theta_{n-1}$. We set

$$
S_{1}(f)=\left\{x \in \mathbb{R}^{n} \mid r=f\left(\theta_{1}, \ldots, \theta_{n-1}\right)\right\}
$$

where $\left(r, \theta_{1}, \ldots, \theta_{n-1}\right)$ are the polar coordinates of $x$. For $\varepsilon$ positive small enough, we introduce the following map

$$
g_{\varepsilon}: S_{1}(f) \rightarrow g_{\varepsilon}\left(S_{1}(f)\right)=S_{2}(f), \quad x \mapsto g_{\varepsilon}(x)=x+\varepsilon n_{x}
$$

where $n_{x}$ is the outward normal to $S_{1}(f)$ at $x$. We denote by $\left(A_{\varepsilon}\right)_{\varepsilon>0}$ the family of annulus shaped open sets in $\mathbb{R}^{n}$ such that $\partial A_{\varepsilon}=S_{1}(f) \cup S_{2}(f)$. Our main result is the following theorem.

THEOREM 1.1. - Assume that $n \geqslant 4$. Let $C$ be any positive constant. Then, there exists $\varepsilon_{0}>0$ such that for any $\varepsilon<\varepsilon_{0}$, the problem $P_{\varepsilon}:-\Delta u_{\varepsilon}=u_{\varepsilon}^{(n+2) /(n-2)}, u_{\varepsilon}>$ 0 in $A_{\varepsilon}, u_{\varepsilon}=0$ on $\partial A_{\varepsilon}$, has no solution such that $\int_{A_{\varepsilon}}\left|\nabla u_{\varepsilon}\right|^{2} \leqslant C$.

Remark 1.2. - We believe the result to be true also for $n=3$ (see Remark 1.4 below).

The proof of Theorem 1.1 is given in two principal steps:

Step 1. We suppose that $P_{\varepsilon}$ has a solution $u_{\varepsilon}$ which satisfies $\int_{A_{\varepsilon}}\left|\nabla u_{\varepsilon}\right|^{2} \leqslant C, C$ being a given constant. We study the asymptotic behavior of $u_{\varepsilon}$ when $\varepsilon$ tends to zero. We prove that $u_{\varepsilon}$ blows up at p points $\left(p \in \mathbb{N}^{*}\right)$, then the location of blow up points is studied. In order to formulate the result of this step, we need to introduce some notations.

We denote by $G_{\varepsilon}$ the Green's function of Laplace operator defined by $\forall x \in A_{\varepsilon}$

$$
-\Delta G_{\varepsilon}(x, .)=c_{n} \delta_{x} \quad \text { in } A_{\varepsilon}, \quad G_{\varepsilon}(x, .)=0 \quad \text { on } \partial A_{\varepsilon}
$$

where $\delta_{x}$ is the Dirac mass at $x$ and $c_{n}=(n-2)$ meas $\left(S^{n-1}\right)$. We denote by $H_{\varepsilon}$ the regular part of $G_{\varepsilon}$, that is,

$$
H_{\varepsilon}\left(x_{1}, x_{2}\right)=\left|x_{1}-x_{2}\right|^{2-n}-G_{\varepsilon}\left(x_{1}, x_{2}\right), \quad \text { for }\left(x_{1}, x_{2}\right) \in A_{\varepsilon} \times A_{\varepsilon} .
$$


For $p \in \mathbb{N}^{*}$ and $x=\left(x_{1}, \ldots, x_{p}\right) \in A_{\varepsilon}^{p}$, we denote by $M=M_{\varepsilon}$ the matrix defined by

$$
M=\left(m_{i j}\right)_{1 \leqslant i, j \leqslant p}, \quad \text { where } m_{i i}=H_{\varepsilon}\left(x_{i}, x_{i}\right), m_{i j}=-G_{\varepsilon}\left(x_{i}, x_{j}\right), i \neq j,
$$

and define $\rho_{\varepsilon}(x)$ as the least eingenvalue of $M(x)\left(\rho_{\varepsilon}(x)=-\infty\right.$ if $x_{i}=x_{j}$ for some $i \neq j)$. For $a \in \mathbb{R}^{n}$ and $\lambda>0, \delta_{(a, \lambda)}$ denotes the function

$$
\delta_{(a, \lambda)}(x)=c_{0} \frac{\lambda^{\frac{n-2}{2}}}{\left(1+\lambda^{2}|x-a|^{2}\right)^{\frac{n-2}{2}}} .
$$

It is well known that if $c_{0}$ is suitably chosen $\left(c_{0}=(n(n-2))^{\frac{n-2}{4}}\right)$ the function $\delta_{(a, \lambda)}$ are the only solutions of equation

$$
-\Delta u=u^{\frac{n+2}{n-2}}, \quad u>0 \quad \text { in } \mathbb{R}^{n}
$$

and they are also the only minimizers for the Sobolev inequality

$$
S=\inf \left\{|\nabla u|_{L^{2}\left(\mathbb{R}^{n}\right)}^{2}|u|_{L^{\frac{2 n}{n-2}\left(\mathbb{R}^{n}\right)}}^{-2} \text {, s.t. } \nabla u \in L^{2}, u \in L^{\frac{2 n}{n-2}}, u \neq 0\right\} .
$$

We also denote by $P_{\varepsilon} \delta_{(a, \lambda)}$ the projection of $\delta_{(a, \lambda)}$ on $H_{0}^{1}\left(A_{\varepsilon}\right)$, that is,

$$
-\Delta P_{\varepsilon} \delta_{(a, \lambda)}=-\Delta \delta_{(a, \lambda)} \text { in } A_{\varepsilon}, P_{\varepsilon} \delta_{(a, \lambda)}=0 \text { on } \partial A_{\varepsilon} .
$$

Lastly, we define on $H_{0}^{1}\left(A_{\varepsilon}\right) \backslash\{0\}$ the functional

$$
J_{\varepsilon}(u)=\frac{\int_{A_{\varepsilon}}|\nabla u|^{2}}{\left(\int_{A_{\varepsilon}}|u|^{\frac{2 n}{n-2}}\right)^{\frac{n-2}{n}}}
$$

whose positive critical points, up a multiplicative constant, are solutions of $P_{\varepsilon}$.

Now we are able to state the main result of step 1.

THEOREM 1.3. - Let $u_{\varepsilon}$ be a solution of problem $P_{\varepsilon}$, assume $\int_{A_{\varepsilon}}\left|\nabla u_{\varepsilon}\right|^{2} \leqslant C$, where $C$ is a positive constant independent of $\varepsilon$. Then, after passing to a subsequence, there exist $p \in \mathbb{N}^{*},\left(a_{1, \varepsilon}, \ldots, a_{p, \varepsilon}\right) \in A_{\varepsilon}^{p},\left(\lambda_{1, \varepsilon}, \ldots, \lambda_{p, \varepsilon}\right) \in\left(\mathbb{R}_{+}^{*}\right)^{p}$ such that:

(i)

$$
\begin{aligned}
& \left|\nabla\left(u_{\varepsilon}-\sum_{i=1}^{p} P_{\varepsilon} \delta_{\left(a_{i, \varepsilon}, \lambda_{i, \varepsilon}\right)}\right)\right|_{L^{2}\left(A_{\varepsilon}\right)} \rightarrow 0, \\
& \quad \lambda_{i, \varepsilon} \rightarrow+\infty, \quad \lambda_{i, \varepsilon} d_{i, \varepsilon} \rightarrow+\infty, \quad \varepsilon_{i j} \rightarrow 0
\end{aligned}
$$

when $\varepsilon \rightarrow 0$, where $d_{i, \varepsilon}=d\left(a_{i, \varepsilon}, \partial A_{\varepsilon}\right)$ and

$$
\varepsilon_{i j}=\left(\frac{\lambda_{i, \varepsilon}}{\lambda_{j, \varepsilon}}+\frac{\lambda_{j, \varepsilon}}{\lambda_{i, \varepsilon}}+\lambda_{i, \varepsilon} \cdot \lambda_{j, \varepsilon}\left|a_{i, \varepsilon}-a_{j, \varepsilon}\right|^{2}\right)^{-\frac{n-2}{2}} .
$$


(ii) Moreover $p \geqslant 2$ and if $n \geqslant 4$, then we have: $\exists k \leqslant p, \exists i_{1}, \ldots, i_{k} \in\{1,2, \ldots, p\}$ such that

$$
d^{n-2} \rho_{\varepsilon}\left(a_{i_{1}, \varepsilon}, \ldots, a_{i_{k}, \varepsilon}\right) \rightarrow 0, \quad d^{n-1} \nabla \rho_{\varepsilon}\left(a_{i_{1}, \varepsilon}, \ldots, a_{i_{k}, \varepsilon}\right) \rightarrow 0
$$

$\forall m, l \in\{1, \ldots, k\}\left|a_{i_{m}, \varepsilon}-a_{i_{l}, \varepsilon}\right| \leqslant C_{0} d$, where $d=\min \left\{d\left(a_{i_{l}, \varepsilon}, \partial A_{\varepsilon}\right) \mid 1 \leqslant l \leqslant k\right\}$ and $C_{0}$ is a positive constant independent of $\varepsilon$.

Remark 1.4. - We believe the result of part (ii) in the Theorem 1.3 to be true for $n=3$. For $n=3$ our method also proves easily $d^{n-2} \rho_{\varepsilon}\left(a_{i_{1}, \varepsilon}, \ldots, a_{i_{k}, \varepsilon}\right) \rightarrow 0$, but for the proof of $d^{n-1} \nabla \rho_{\varepsilon}\left(a_{i_{1}, \varepsilon}, \ldots, a_{i_{k}, \varepsilon}\right) \rightarrow 0$ we need a more careful estimates of the rests in Propositions 3.2 and 3.3 below.

The main ingredients of the proof of the Theorem 1.3 are a fine blow-up analysis, on the one hand, and a very delicate expansion of $\nabla J$ near infinity, on the other hand.

Step 2. We prove the following result.

TheOREM 1.5. - For $n \geqslant 3$, let $C_{0}>0$ and let $\left(x_{1}, x_{2}, \ldots, x_{k}\right) \in A_{\varepsilon}^{k}$ such that

$$
d^{n-2} \rho_{\varepsilon}\left(x_{1}, \ldots, x_{k}\right) \rightarrow 0 \quad \text { when } \varepsilon \rightarrow 0 \text { and }\left|x_{i}-x_{j}\right| \leqslant C_{0} d, \forall i, j,
$$

where $d=\min \left\{d\left(x_{i}, \partial A_{\varepsilon}\right) \mid 1 \leqslant i \leqslant k\right\}$. Then

$$
d^{n-1} \nabla \rho_{\varepsilon}\left(x_{1}, \ldots, x_{k}\right) \not \rightarrow 0 \quad \text { when } \varepsilon \rightarrow 0 .
$$

We notice that Theorem 1.1 is an easy consequence of Theorems 1.3 and 1.5.

The remainder of the present paper is organised as follows. Section 2 is devoted to the proof of the first part of Theorem 1.3, while the second part of Theorem 1.3 is proved in Section 3. In Section 4 we give the proof of Theorem 1.5. Lastly, we give in Appendix A some technical lemmas needed in Section 3.

\section{Asymptotic behavior of solutions with bounded energy}

In this section, we will study the asymptotic behavior of solutions $u_{\varepsilon}$ of $P_{\varepsilon}$ when $\varepsilon$ is small enough and their energy is bounded. Thus, in the remainder, we assume that $\int_{A_{\varepsilon}}\left|\nabla u_{\varepsilon}\right|^{2} \leqslant C$, where $C$ is a positive constant independent of $\varepsilon$. We begin by proving the following lemma.

LEMMA 2.1. - We have the following claim

$$
\int_{A_{\varepsilon}}\left|\nabla u_{\varepsilon}\right|^{2} \nrightarrow 0, \quad M_{\varepsilon} \rightarrow+\infty, \quad \text { when } \varepsilon \rightarrow 0, \text { where } M_{\varepsilon}=\left|u_{\varepsilon}\right|_{L^{\infty}\left(A_{\varepsilon}\right)} .
$$

Proof. - On the one hand, since $u_{\varepsilon}$ is a solution of $P_{\varepsilon}$, we have

$$
\int_{A_{\varepsilon}}\left|\nabla u_{\varepsilon}\right|^{2}=\int_{A_{\varepsilon}} u_{\varepsilon}^{\frac{2 n}{n-2}}
$$


On the other hand, we have

$$
\left(\int_{A_{\varepsilon}}\left|u_{\varepsilon}\right|^{\frac{2 n}{n-2}}\right)^{\frac{n-2}{n}} \leqslant \frac{1}{S} \int_{A_{\varepsilon}}\left|\nabla u_{\varepsilon}\right|^{2}
$$

where $S$ denotes the Sobolev constant defined in (1.6). Thus

$$
S_{n}=S^{\frac{n}{2}} \leqslant \int_{A_{\varepsilon}}\left|\nabla u_{\varepsilon}\right|^{2}=\int_{A_{\varepsilon}} u_{\varepsilon}^{\frac{2 n}{n-2}} \leqslant c \varepsilon M_{\varepsilon}^{\frac{2 n}{n-2}}
$$

and our lemma follows.

LEMmA 2.2. - There exists a positive constant $c$ such that for $\varepsilon$ small enough, we have

$$
\varepsilon M_{\varepsilon}^{\frac{2}{n-2}} \geqslant c, \quad \text { where } M_{\varepsilon}=\left|u_{\varepsilon}\right|_{L^{\infty}\left(A_{\varepsilon}\right)} .
$$

Proof. - On the one hand, we have

$$
\int_{A_{\varepsilon}}\left|\nabla u_{\varepsilon}\right|^{2}=\int_{A_{\varepsilon}} u_{\varepsilon}^{\frac{2 n}{n-2}} \leqslant M_{\varepsilon}^{\frac{4}{n-2}} \int_{A_{\varepsilon}} u_{\varepsilon}^{2}(x) \mathrm{d} x .
$$

On the other hand, we have

$$
\int_{A_{\varepsilon}} u_{\varepsilon}^{2}(x) \mathrm{d} x=\varepsilon^{n} \int_{B_{\varepsilon}} v_{\varepsilon}^{2}(X) \mathrm{d} X
$$

where $v_{\varepsilon}(X)=u_{\varepsilon}(\varepsilon X)$ and where $B_{\varepsilon}=\varphi\left(A_{\varepsilon}\right)$, with $\varphi: x \mapsto \varphi(x)=\varepsilon^{-1} x$. Observe that

$$
\varepsilon^{n} \int_{B_{\varepsilon}} v_{\varepsilon}^{2}(X) \mathrm{d} X \leqslant \frac{\varepsilon^{n}}{c_{\varepsilon}} \int_{B_{\varepsilon}}\left|\nabla v_{\varepsilon}(X)\right|^{2} \mathrm{~d} X=\frac{\varepsilon^{2}}{c_{\varepsilon}} \int_{A_{\varepsilon}}\left|\nabla u_{\varepsilon}(x)\right|^{2} \mathrm{~d} x .
$$

Thus

$$
c_{\varepsilon} \leqslant \varepsilon^{2} M_{\varepsilon}^{\frac{4}{n-2}} .
$$

According to Lin [12], we have $\lim _{\varepsilon \rightarrow 0} c_{\varepsilon}=c>0$, therefore our lemma follows.

Now let $\widetilde{A}_{\varepsilon}=M_{\varepsilon}^{\frac{2}{n-2}}\left(A_{\varepsilon}-a_{1, \varepsilon}\right)$, where $a_{1, \varepsilon} \in A_{\varepsilon}$ such that $M_{\varepsilon}=u_{\varepsilon}\left(a_{1, \varepsilon}\right)$, and we denote by $v_{\varepsilon}$ the function defined on $\widetilde{A}_{\varepsilon}$ by

$$
v_{\varepsilon}(X)=M_{\varepsilon}^{-1} u_{\varepsilon}\left(a_{1, \varepsilon}+M_{\varepsilon}^{\frac{-2}{n-2}} X\right)
$$

It is easy to see that $v_{\varepsilon}$ satisfies

$$
\begin{cases}-\Delta v_{\varepsilon}=v_{\varepsilon}^{\frac{n+2}{n-2}}, \quad 0<v_{\varepsilon} \leqslant 1 & \text { in } \widetilde{A}_{\varepsilon} \\ v_{\varepsilon}(0)=1, \quad v_{\varepsilon}=0 & \text { on } \partial \widetilde{A}_{\varepsilon} .\end{cases}
$$


Observe that

$$
\int_{\widetilde{A}_{\varepsilon}}\left|\nabla v_{\varepsilon}\right|^{2}=\int_{A_{\varepsilon}}\left|\nabla u_{\varepsilon}\right|^{2}=\int_{\widetilde{A}_{\varepsilon}} v_{\varepsilon}^{\frac{2 n}{n-2}}=\int_{A_{\varepsilon}} u_{\varepsilon}^{\frac{2 n}{n-2}} \leqslant C .
$$

Let us prove the following lemma.

LEMMA 2.3. - We have the following claim

$$
M_{\varepsilon}^{\frac{2}{n-2}} d\left(a_{1, \varepsilon}, \partial A_{\varepsilon}\right) \rightarrow+\infty, \quad \text { when } \varepsilon \rightarrow 0 .
$$

Proof. - Let $l=\lim _{\varepsilon \rightarrow 0} M_{\varepsilon}^{2 /(n-2)} d\left(a_{1, \varepsilon}, \partial A_{\varepsilon}\right)$. First we will prove $l \neq 0$. A similar result has been proved by Harrabi, Rebhi and Selmi [11]. We will adapt their proof to our case. Let $\bar{a}_{1, \varepsilon} \in \partial A_{\varepsilon}$ such that $d_{\varepsilon}:=d\left(a_{1, \varepsilon}, \partial A_{\varepsilon}\right)=\left|\bar{a}_{1, \varepsilon}-a_{1, \varepsilon}\right|$. We may assume without loss of generality that the unit outward normal to $\partial A_{\varepsilon}$ at $\bar{a}_{1, \varepsilon}$ is $e_{n}$, where $e_{n}$ is the last element of a canonical basic of $\mathbb{R}^{n}$. We see that $d_{\varepsilon}=a_{1, \varepsilon} \cdot e_{n}=a_{1, \varepsilon}^{n}$, where $a_{1, \varepsilon}=\left(a_{1, \varepsilon}^{1}, \ldots, a_{1, \varepsilon}^{n}\right) . v_{\varepsilon}$ is well defined in

$$
B\left(0,(2 / 3) \varepsilon M_{\varepsilon}^{2 /(n-2)}\right) \cap\left\{\left(x^{1}, \ldots, x^{n}\right) /-d_{\varepsilon} M_{\varepsilon}^{2 /(n-2)}<x^{n}<\left(2 \varepsilon / 3-d_{\varepsilon}\right) M_{\varepsilon}^{2 /(n-2)}\right\} .
$$

Let $z_{\varepsilon}=\left(0, \ldots,-d_{\varepsilon} M_{\varepsilon}^{2 /(n-2)}\right)$ and let $\tilde{v}_{\varepsilon}(X)=v_{\varepsilon}\left(X+z_{\varepsilon}\right)$, for

$$
X \in B\left(-z_{\varepsilon},(2 \varepsilon / 3) M_{\varepsilon}^{2 /(n-2)}\right) \cap\left\{0<x^{n}<(2 \varepsilon / 3) M_{\varepsilon}^{2 /(n-2)}\right\} .
$$

We suppose, arguing by contradiction, that $\left|z_{\varepsilon}\right|=l_{\varepsilon} \rightarrow 0$ as $\varepsilon \rightarrow 0$. Then $-z_{\varepsilon} \in$ $B^{+}(0, \theta)=B(0, \theta) \cap\left\{\left(x^{1}, \ldots, x^{n}\right) / x^{n}>0\right\}$, where $\theta$ is a fixed positive real small enough choosen below. Let $R>0$ be such that

$$
B^{+}(0, \theta) \subset B^{+}(0, R) \subset B\left(-z_{\varepsilon},(2 / 3) \varepsilon M_{\varepsilon}^{\frac{2}{n-2}}\right) \cap\left\{0<x^{n}<(2 / 3) \varepsilon M_{\varepsilon}^{\frac{2}{n-2}}\right\}
$$

for $\varepsilon$ small enough. We consider the following equation

$$
\begin{cases}-\Delta \omega_{\varepsilon}=0 & \text { in } B^{+}(0, R) \\ \omega_{\varepsilon}=\tilde{v}_{\varepsilon} & \text { on } \partial B^{+}(0, R)\end{cases}
$$

Then $\omega_{\varepsilon} \in C^{2}\left(B^{+}(0, R)\right) \cap C^{0}\left(\overline{B^{+}(0, R)}\right)$. We derive that

$$
\left|\tilde{v}_{\varepsilon}-\omega_{\varepsilon}\right|_{W^{2, q}\left(B^{+}(0, R)\right)} \leqslant c, \quad \forall q<\infty .
$$

In particular, $\operatorname{grad}\left(\tilde{v}_{\varepsilon}-\omega_{\varepsilon}\right)$ is bounded in $B^{+}(0, R)$, and we have

$$
\frac{\partial \tilde{v}_{\varepsilon}}{\partial x^{n}}<c+\frac{\partial \omega_{\varepsilon}}{\partial x^{n}} .
$$

Observe that

$$
\omega_{\varepsilon}(x)=-\int_{\partial B^{+}(0, R)} \frac{\partial G_{B^{+}}}{\partial v} \omega_{\varepsilon}(y) \mathrm{d} y \quad \text { in } B^{+}(0, \theta) .
$$


Thus

$$
\frac{\partial \omega_{\varepsilon}}{\partial x^{n}}(x)=-\int_{\partial B^{+}(0, R)} \frac{\partial}{\partial x^{n}} \frac{\partial G_{B^{+}}}{\partial v} \omega_{\varepsilon}(y) \mathrm{d} y .
$$

According to Lemma 2.6 [10], we have

$\exists \theta>0, \exists c>0 \quad 0<-\frac{\partial}{\partial x^{n}} \frac{\partial G_{B^{+}}}{\partial v}(x, y) \leqslant c, \quad \forall y \in \partial B^{+}(0, R),\left|x^{\prime}\right|<\frac{1}{2}, 0<x^{n}<\theta$.

Since $\omega_{\varepsilon} \leqslant 1$, we derive that $\partial \omega_{\varepsilon} / \partial x^{n}$ is bounded for $0<x^{n}<\theta$. Thus $\partial \tilde{v}_{\varepsilon} / \partial x^{n}<c^{\prime}$. Let $f_{\varepsilon}(t)=\tilde{v}_{\varepsilon}\left(t e_{n}\right)$, then we have

$$
f_{\varepsilon}\left(l_{\varepsilon}\right)-f_{\varepsilon}(0)=f_{\varepsilon}^{\prime}\left(c_{\varepsilon}\right) l_{\varepsilon}=\frac{\partial \tilde{v}_{\varepsilon}}{\partial x^{n}}\left(c_{\varepsilon} e_{n}\right) l_{\varepsilon} \leqslant c^{\prime} l_{\varepsilon}
$$

where $l_{\varepsilon}=M_{\varepsilon}^{\frac{2}{n-2}} d_{\varepsilon}$. Hence $f_{\varepsilon}\left(l_{\varepsilon}\right)-f_{\varepsilon}(0)=\tilde{v}_{\varepsilon}(0)-\tilde{v}_{\varepsilon}\left(z_{\varepsilon}\right)=1 \leqslant c^{\prime} l_{\varepsilon}$ which contradicts the assumption $l_{\varepsilon} \rightarrow 0$. We derive that $l \neq 0$. We suppose, arguing by contradiction, $l<\infty$. Then it follows from (2.2) and standard elliptic theories that there exists some positive function $v$, such that (after passing to subsequence), $v_{\varepsilon} \rightarrow v$ in $C_{\mathrm{loc}}^{1}(\Omega)$, where $\Omega$ is a half space or a strip of $\mathbb{R}^{n}$, and $v$ satisfies

$$
\begin{cases}-\Delta v=v^{\frac{n+2}{n-2}}, \quad v>0 & \text { in } \Omega, \\ v(0)=1, \quad v=0 & \text { on } \partial \Omega .\end{cases}
$$

But if $\Omega$ is a half space or a strip of $\mathbb{R}^{n}$, by Pohozaev Identity (see Theorem III.1.3 [15]), then $v$ must vanish identically. Thus we derive a contradiction and our lemma follows.

From Lemma 2.3, we derive that there exists some positive function $v$, such that (after passing to a subsequence), $v_{\varepsilon} \rightarrow v$ in $C_{\text {loc }}^{1}\left(\mathbb{R}^{n}\right)$, and $v$ satisfies

$$
\left\{\begin{array}{l}
-\Delta v=v^{\frac{n+2}{n-2}}, \quad v>0 \quad \text { in } \mathbb{R}^{n}, \\
v(0)=1, \quad \nabla v(0)=0 .
\end{array}\right.
$$

It follows from Cafferalli, Gidas and Spruck [7]

$$
v(X)=\delta_{\left(0, \alpha_{n}\right)}(X), \quad \text { with } \alpha_{n}=(n(n-2))^{-1 / 2} .
$$

Hence

$$
M_{\varepsilon}^{-1} u_{\varepsilon}\left(a_{1, \varepsilon}+M_{\varepsilon}^{\frac{-2}{n-2}} X\right)-\delta_{\left(0, \alpha_{n}\right)}(X) \rightarrow 0 \quad \text { in } C_{\mathrm{loc}}^{1}\left(\mathbb{R}^{n}\right) \text {, when } \varepsilon \rightarrow 0 .
$$

Observe that

$$
M_{\varepsilon}^{-1} u_{\varepsilon}\left(a_{1, \varepsilon}+M_{\varepsilon}^{\frac{-2}{n-2}} X\right)-\delta_{\left(0, \alpha_{n}\right)}(X)=M_{\varepsilon}^{-1}\left(u_{\varepsilon}(x)-\delta_{\left(a_{1, \varepsilon}, \lambda_{1, \varepsilon}\right)}(x)\right)
$$

where $\lambda_{1, \varepsilon}=\alpha_{n} M_{\varepsilon}^{\frac{2}{n-2}}$. 
In the sequel, we denote by $u_{\varepsilon}^{1}$ the function defined on $A_{\varepsilon}$ by

$$
u_{\varepsilon}^{1}(x)=u_{\varepsilon}(x)-P_{\varepsilon} \delta_{\left(a_{1, \varepsilon}, \lambda_{1, \varepsilon}\right)}(x) .
$$

Notice that $\lambda_{1, \varepsilon} \rightarrow+\infty$ and $\lambda_{1, \varepsilon} d\left(a_{1, \varepsilon}, \partial A_{\varepsilon}\right) \rightarrow+\infty$ when $\varepsilon \rightarrow 0$.

LEMMA 2.4. - Let $u_{\varepsilon}^{1}$ be defined by (2.4). Then we have

(i) $-\Delta u_{\varepsilon}^{1}=\left|u_{\varepsilon}^{1}\right|^{\frac{4}{n-2}} u_{\varepsilon}^{1}+g_{\varepsilon}$, with $\left|g_{\varepsilon}\right|_{H_{\left(A_{\varepsilon}\right)}^{-1}} \rightarrow 0$, when $\varepsilon \rightarrow 0$.

(ii) $\int_{A_{\varepsilon}}\left|\nabla u_{\varepsilon}^{1}\right|^{2}=\int_{A_{\varepsilon}}\left|\nabla u_{\varepsilon}\right|^{2}-S_{n}+\mathrm{o}(1)$.

(iii) $\int_{A_{\varepsilon}}\left|u_{\varepsilon}^{1}\right|^{\frac{2 n}{n-2}}=\int_{A_{\varepsilon}}\left|u_{\varepsilon}\right|^{\frac{2 n}{n-2}}-S_{n}+o(1)$ where $S_{n}=S^{\frac{n}{2}}$.

Proof. -

(i) We have

$$
-\Delta u_{\varepsilon}^{1}=-\Delta u_{\varepsilon}+\Delta P_{\varepsilon} \delta_{\left(a_{1, \varepsilon}, \lambda_{1, \varepsilon}\right)}=u_{\varepsilon}^{\frac{n+2}{n-2}}-\delta_{\left(a_{1, \varepsilon}, \lambda_{1, \varepsilon}\right)}^{\frac{n+2}{n-2}}=\left|u_{\varepsilon}^{1}\right|^{\frac{4}{n-2}} u_{\varepsilon}^{1}+g_{\varepsilon}
$$

where

$$
g_{\varepsilon}=u_{\varepsilon}^{\frac{n+2}{n-2}}-\delta_{\left(a_{1, \varepsilon}, \lambda_{1, \varepsilon}\right)}^{\frac{n+2}{n-2}}-\left|u_{\varepsilon}^{1}\right|^{\frac{4}{n-2}} u_{\varepsilon}^{1} .
$$

Observe that

$$
g_{\varepsilon}=\mathrm{O}\left(\left|P_{\varepsilon} \delta\right|^{\frac{4}{n-2}}\left|u_{\varepsilon}-P_{\varepsilon} \delta\right|+\left|u_{\varepsilon}-P_{\varepsilon} \delta\right|^{\frac{4}{n-2}} P_{\varepsilon} \delta\right)+\mathrm{O}\left(\delta_{\varepsilon}^{\frac{4}{n-2}}\left(\delta_{\varepsilon}-P_{\varepsilon} \delta\right)\right)
$$

where $P_{\varepsilon} \delta=P_{\varepsilon} \delta_{\left(a_{1, \varepsilon}, \lambda_{1, \varepsilon}\right)}$ and $\delta_{\varepsilon}=\delta_{\left(a_{1, \varepsilon}, \lambda_{1, \varepsilon}\right)}$. Since $L^{\frac{2 n}{n+2}} \hookrightarrow H^{-1}$, it is sufficient to prove that

$$
\int_{A_{\varepsilon}} \delta_{\varepsilon}^{\frac{8 n}{n^{2}-4}}\left|u_{\varepsilon}-P_{\varepsilon} \delta\right|^{\frac{2 n}{n+2}} \rightarrow 0 \quad \text { and } \quad \int_{A_{\varepsilon}} \delta_{\varepsilon}^{\frac{2 n}{n+2}}\left|u_{\varepsilon}-P_{\varepsilon} \delta\right|^{\frac{8 n}{n^{2}-4}} \rightarrow 0, \quad \text { when } \varepsilon \rightarrow 0 .
$$

Observe that

$$
\begin{aligned}
& \int_{A_{\varepsilon}} \delta_{\varepsilon}^{\frac{8 n}{n^{2}-4}}\left|u_{\varepsilon}-P_{\varepsilon} \delta\right|^{\frac{2 n}{n+2}} \leqslant c \int_{A_{\varepsilon}} \delta_{\varepsilon}^{\frac{8 n}{n^{2}-4}}\left|u_{\varepsilon}-\delta_{\varepsilon} \frac{2 n}{n^{\frac{2 n}{n+2}}}+c \int_{A_{\varepsilon}} \delta_{\varepsilon}^{\frac{8 n}{n^{2}-4}}\right| \delta_{\varepsilon}-\left.P_{\varepsilon} \delta\right|^{\frac{2 n}{n^{n+2}}} \\
& \leqslant c \int_{\widetilde{A}_{\varepsilon}} \delta_{\left(0, \alpha_{n}\right)}^{\frac{8 n}{n^{2}-4}}(X)\left|v_{\varepsilon}(X)-M_{\varepsilon}^{-1} \delta_{\varepsilon}\left(a_{1, \varepsilon}+M_{\varepsilon}^{\frac{-2}{n-2}} X\right)\right|^{\frac{2 n}{n+2}} \mathrm{~d} X \\
&+\mathrm{O}\left(\left|\delta_{\varepsilon}-P_{\varepsilon} \delta\right|^{\frac{2 n}{n+2}}\right) \\
&= c \int_{B(0, R)}+c \int_{\widetilde{A}_{\varepsilon} \backslash B(0, R)}^{\frac{2 n}{n-2}}+\mathrm{o}(1)
\end{aligned}
$$

where $R$ is a large enough positive constant such that $\int_{\mathbb{R}^{n} \backslash B(0, R)} \delta_{\left(0, \alpha_{n}\right)}^{\frac{2 n}{n-2}}=\mathrm{o}(1)$.

Now we are going to estimate the 2 nd integral 


$$
\begin{aligned}
& \int_{\widetilde{A}_{\varepsilon} \backslash B(0, R)} \delta_{\left(0, \alpha_{n}\right)}^{\frac{8 n}{n^{2}-4}}(X)\left|v_{\varepsilon}(X)-\delta_{\left(0, \alpha_{n}\right)}(X)\right|^{\frac{2 n}{n+2}} \mathrm{~d} X \\
& \leqslant\left(\int_{\widetilde{A}_{\mathcal{E}} \backslash B(0, R)} \delta_{\left(0, \alpha_{n}\right)}^{\frac{2 n}{n-2}}(X)\right)^{\frac{4}{n+2}}\left(\int_{\widetilde{A}_{\varepsilon} \backslash B(0, R)}\left|v_{\varepsilon}(X)-\delta_{\left(0, \alpha_{n}\right)}(X)\right|^{\frac{2 n}{n-2}}\right)^{\frac{n-2}{n+2}} \\
& \quad \leqslant c_{1}\left(\int_{\mathbb{R}^{n} \backslash B(0, R)} \delta_{\left(0, \alpha_{n}\right)}^{\frac{2 n}{n-2}}(X)\right)^{\frac{4}{n+2}}
\end{aligned}
$$

indeed $\int_{\widetilde{A}_{\varepsilon}} v^{\frac{2 n}{n-2}} \leqslant C$. For the first integral, we have

$$
\int_{B(0, R)} \delta_{\left(0, \alpha_{n}\right)}^{\frac{8 n}{n^{2}-4}}(X)\left|v_{\varepsilon}(X)-\delta_{\left(0, \alpha_{n}\right)}(X)\right|^{\frac{2 n}{n+2}} \mathrm{~d} X \leqslant C \int_{B(0, R)}\left|v_{\varepsilon}(X)-\delta_{\left(0, \alpha_{n}\right)}(X)\right|^{\frac{2 n}{n+2}} \mathrm{~d} X \rightarrow 0,
$$

when $\varepsilon \rightarrow 0$, indeed $v_{\varepsilon}-\delta_{\left(0, \alpha_{n}\right)} \rightarrow 0$ in $C_{\text {loc }}^{1}\left(\mathbb{R}^{n}\right)$. In the same way, we prove that

$$
\int_{A_{\varepsilon}} \delta_{\varepsilon}^{\frac{2 n}{n+2}}\left|u_{\varepsilon}-P_{\varepsilon} \delta\right|^{\frac{8 n}{n^{2}-4}} \rightarrow 0, \quad \text { when } \varepsilon \rightarrow 0 .
$$

(ii) We also have

$$
\int_{A_{\varepsilon}}\left|\nabla u_{\varepsilon}^{1}\right|^{2}=\int_{A_{\varepsilon}}\left|\nabla u_{\varepsilon}\right|^{2}+\int_{A_{\varepsilon}}\left|\nabla P_{\varepsilon} \delta\right|^{2}-2 \int_{A_{\varepsilon}} \nabla u_{\varepsilon} \nabla P_{\varepsilon} \delta
$$

Observe that

$$
\begin{aligned}
\int_{A_{\varepsilon}}\left|\nabla P_{\varepsilon} \delta\right|^{2} & =\int_{A_{\varepsilon}} \delta_{\varepsilon}^{\frac{n+2}{n-2}} P_{\varepsilon} \delta=\int_{A_{\varepsilon}} \delta_{\varepsilon}^{\frac{2 n}{n-2}}-\int_{A_{\varepsilon}} \delta_{\varepsilon}^{\frac{n+2}{n-2}}\left(\delta_{\varepsilon}-P_{\varepsilon} \delta\right) \\
& =\int_{\widetilde{A}_{\varepsilon}} \delta_{\left(0, \alpha_{n}\right)}^{\frac{2 n}{n-2}}-\int_{A_{\varepsilon}} \delta_{\varepsilon}^{\frac{n+2}{n-2}}\left(\delta_{\varepsilon}-P_{\varepsilon} \delta\right) \\
& =S_{n}-\int_{\mathbb{R}^{n} \backslash \widetilde{A}_{\varepsilon}} \delta_{\left(0, \alpha_{n}\right)}^{\frac{2 n}{n-2}}-\int_{A_{\varepsilon}} \delta_{\varepsilon}^{\frac{n+2}{n-2}}\left(\delta_{\varepsilon}-P_{\varepsilon} \delta\right) .
\end{aligned}
$$

For the 2nd integral, we have

$$
\int_{A_{\varepsilon}} \delta_{\varepsilon}^{\frac{n+2}{n-2}}\left(\delta_{\varepsilon}-P_{\varepsilon} \delta\right) \leqslant C\left|\delta_{\varepsilon}-P_{\varepsilon} \delta\right|_{L^{\frac{2 n}{n-2}\left(A_{\varepsilon}\right)}} \leqslant c\left(\lambda_{1, \varepsilon} d_{1, \varepsilon}\right)^{\frac{2-n}{2}} \rightarrow 0, \quad \text { when } \varepsilon \rightarrow 0
$$

where $d_{1, \varepsilon}=d\left(a_{1, \varepsilon}, \partial A_{\varepsilon}\right)$.

For the first integral, we have

$$
\int_{\mathbb{R}^{n} \backslash \widetilde{A}_{\varepsilon}} \delta_{\left(0, \alpha_{n}\right)}^{\frac{2 n}{n-2}}=\mathrm{o}(1)
$$


indeed $\widetilde{A}_{\varepsilon} \rightarrow \mathbb{R}^{n}$ and $\delta_{\left(0, \alpha_{n}\right)} \in L^{\frac{2 n}{n-2}}\left(\mathbb{R}^{n}\right)$. Then

$$
\int_{A_{\varepsilon}}\left|\nabla P_{\varepsilon} \delta\right|^{2}=S_{n}+\mathrm{o}(1)
$$

We also have

$$
\int_{A_{\varepsilon}} \nabla u_{\varepsilon} \nabla P_{\varepsilon} \delta=\int_{A_{\varepsilon}} \nabla\left(u_{\varepsilon}-P_{\varepsilon} \delta\right) \nabla P_{\varepsilon} \delta+\int_{A_{\varepsilon}}\left|\nabla P_{\varepsilon} \delta\right|^{2} .
$$

Observe that

$$
\begin{aligned}
\int_{A_{\varepsilon}} \nabla\left(u_{\varepsilon}-P_{\varepsilon} \delta\right) \nabla P_{\varepsilon} \delta & =\int_{A_{\varepsilon}}\left(u_{\varepsilon}-P_{\varepsilon} \delta\right) \delta_{\varepsilon}^{\frac{n+2}{n-2}} \\
& =\int_{A_{\varepsilon}}\left(u_{\varepsilon}-\delta_{\varepsilon}\right) \delta_{\varepsilon}^{\frac{n+2}{n-2}}+\int_{A_{\varepsilon}}\left(\delta_{\varepsilon}-P_{\varepsilon} \delta\right) \delta_{\varepsilon}^{\frac{n+2}{n-2}} \\
& =\int_{\widetilde{A}_{\varepsilon}}\left(v_{\varepsilon}-\delta_{\left(0, \alpha_{n}\right)}\right) \delta_{\left(0, \alpha_{n}\right)}^{\frac{n+2}{n-2}}+o(1) \\
& \leqslant \int_{B(0, R)}\left(v_{\varepsilon}-\delta_{\left(0, \alpha_{n}\right)}\right) \delta_{\left(0, \alpha_{n}\right)}^{\frac{n+2}{n-2}}+\int_{\mathbb{R}^{n} \backslash B(0, R)}\left(v_{\varepsilon}-\delta_{\left(0, \alpha_{n}\right)}\right) \delta_{\left(0, \alpha_{n}\right)}^{\frac{n+2}{n-2}}+o(1) .
\end{aligned}
$$

Notice that, on the one hand

$$
\int_{\mathbb{R}^{n} \backslash B(0, R)}\left(v_{\varepsilon}-\delta_{\left(0, \alpha_{n}\right)}\right) \delta_{\left(0, \alpha_{n}\right)}^{\frac{n+2}{n-2}} \leqslant C\left(\int_{\mathbb{R}^{n} \backslash B(0, R)} \delta_{\left(0, \alpha_{n}\right)}^{\frac{2 n}{n-2}}\right)^{\frac{n+2}{2 n}}=\mathrm{o}(1) .
$$

On the other hand

$$
\int_{B(0, R)}\left(v_{\varepsilon}-\delta_{\left(0, \alpha_{n}\right)}\right) \delta_{\left(0, \alpha_{n}\right)}^{\frac{n+2}{n-2}}=\mathrm{o}(1), \quad \text { since } v_{\varepsilon} \rightarrow \delta_{\left(0, \alpha_{n}\right)} \text { in } C_{\mathrm{loc}}^{1}\left(\mathbb{R}^{n}\right) .
$$

Then

$$
\int_{A_{\varepsilon}} \nabla u_{\varepsilon} \nabla P_{\varepsilon} \delta=S_{n}+\mathrm{o}(1) .
$$

Thus (ii) of Lemma 2.4 follows.

(iii) The proof of (iii) in Lemma 2.4 is similar to the proof of (ii), so we will omit it.

Now, we distinguish two cases

(i) $\int_{A_{\varepsilon}}\left|\nabla u_{\varepsilon}^{1}\right|^{2} \rightarrow 0$ when $\varepsilon \rightarrow 0$.

(ii) $\int_{A_{\varepsilon}}\left|\nabla u_{\varepsilon}^{1}\right|^{2} \nrightarrow \rightarrow 0$ when $\varepsilon \rightarrow 0$.

If $\int_{A_{\varepsilon}}\left|\nabla u_{\varepsilon}^{1}\right|^{2} \rightarrow 0$, the proof of (i) (Theorem 1.3) is finished. 
In the sequel, we consider the second case, that is $\int_{A_{\varepsilon}}\left|\nabla u_{\varepsilon}^{1}\right|^{2} \nrightarrow 0$, when $\varepsilon \rightarrow 0$ and we are going to look for a second point of blow up of $u_{\varepsilon}$.

In order to simplify the notations, in remainder we often omit the index $\varepsilon$ of $a_{\varepsilon}$ and $\lambda_{\varepsilon}$.

Let us introduce the following notations

$$
\begin{gathered}
u_{\varepsilon}\left(a_{2}\right):=\lambda_{2}^{\frac{n-2}{2}}=\max _{\left(A_{\varepsilon} \backslash B\left(a_{1}, \varepsilon\right)\right)} u_{\varepsilon}(x), \\
h_{\varepsilon}=\max _{B\left(a_{1}, 2 \varepsilon\right)}\left|x-a_{1}\right|^{\frac{n-2}{2}} u_{\varepsilon}(x)=\left|a_{1}-a_{3}\right|^{\frac{n-2}{2}} u_{\varepsilon}\left(a_{3}\right)=\left|a_{1}-a_{3}\right|^{\frac{n-2}{2}} \lambda_{3}^{\frac{n-2}{2}} .
\end{gathered}
$$

We distinguish two cases.

Case $1 . h_{\varepsilon} \rightarrow+\infty$ when $\varepsilon \rightarrow 0$.

Case 2. $h_{\varepsilon} \leqslant c$, when $\varepsilon \rightarrow 0$.

Now we study the first case, that is $h_{\varepsilon} \rightarrow \infty$ when $\varepsilon \rightarrow 0$. Let

$$
\lambda_{4}=\max \left(\lambda_{2}, \lambda_{3}\right):=u_{\varepsilon}^{\frac{2}{n-2}}\left(a_{4}\right) .
$$

For $X \in B\left(0, \frac{\lambda_{4}}{2}\left|a_{1}-a_{4}\right|\right) \cap D_{\varepsilon}$, we set

$$
w_{\varepsilon}(X)=\lambda_{4}^{\frac{-(n-2)}{2}} u_{\varepsilon}\left(a_{4}+\lambda_{4}^{-1} X\right), \quad \text { with } D_{\varepsilon}=\lambda_{4}\left(A_{\varepsilon}-a_{4}\right) .
$$

It is easy to check the following claims

$$
\lambda_{4}\left|a_{1}-a_{4}\right| \geqslant(1 / 2) \lambda_{3}\left|a_{1}-a_{3}\right|, \quad \text { and } \quad \lambda_{4} \varepsilon \geqslant(1 / 2) \lambda_{3}\left|a_{1}-a_{3}\right| .
$$

Thus

$$
\lambda_{4}\left|a_{1}-a_{4}\right| \rightarrow+\infty \quad \text { and } \quad \lambda_{4} \varepsilon \rightarrow+\infty \quad \text { as } \varepsilon \rightarrow 0 .
$$

We also have

$$
w_{\varepsilon}(X) \leqslant c, \quad \forall X \in B\left(0,(1 / 2) \lambda_{4}\left|a_{1}-a_{4}\right|\right) \cap D_{\varepsilon} .
$$

As in Lemma 2.3, we can prove

$$
\lambda_{4} d\left(a_{4}, \partial A_{\varepsilon}\right) \rightarrow+\infty, \quad \text { as } \varepsilon \rightarrow 0 .
$$

Thus, there exist $b \in \mathbb{R}^{n}$ and $\lambda>0$ such that $w_{\varepsilon} \rightarrow \delta_{(b, \lambda)}$ in $C_{\mathrm{loc}}^{1}\left(\mathbb{R}^{n}\right)$. Therefore we have found a second point of blow up $\bar{a}_{2}$ of $u_{\varepsilon}$ with the concentration $\bar{\lambda}_{2}$ in this case $\left(\bar{a}_{2}=a_{4}+b / \lambda_{4}\right.$ and $\left.\bar{\lambda}_{2}=\lambda \lambda_{4}\right)$.

Next we study the second case, that is $h_{\varepsilon}$ remains bounded when $\varepsilon \rightarrow 0$, where $h_{\varepsilon}$ is defined in (2.6). In this case we consider two subcases.

(i) $\int_{B\left(a_{1}, 2 \varepsilon\right)}\left|u_{\varepsilon}^{1}\right|^{\frac{2 n}{n-2}} \rightarrow 0$ as $\varepsilon \rightarrow 0$.

(ii) $\int_{B\left(a_{1}, 2 \varepsilon\right)}\left|u_{\varepsilon}^{1}\right|^{\frac{2 n}{n-2}} \nrightarrow 0$ as $\varepsilon \rightarrow 0$. 
Let us consider the first case, that is $h_{\varepsilon}$ remains bounded and $\int_{B\left(a_{1}, 2 \varepsilon\right)}\left|u_{\varepsilon}^{1}\right|^{\frac{2 n}{n-2}} \rightarrow 0$ as $\varepsilon \rightarrow 0$. Thus there exists $c>0$ such that

$$
0<c \leqslant \int_{A_{\varepsilon} \backslash B\left(a_{1}, 2 \varepsilon\right)}\left|u_{\varepsilon}^{1}\right|^{\frac{2 n}{n-2}} \leqslant c \lambda_{2}^{2} \int_{A_{\varepsilon}} u_{\varepsilon}^{2} \leqslant c\left(\varepsilon \lambda_{2}\right)^{2} .
$$

Hence, there exists $\bar{c}>0$ such that

$$
\lambda_{2}\left|a_{1}-a_{2}\right| \geqslant \lambda_{2} \varepsilon \geqslant 2 \bar{c} .
$$

Now, for $X \in E_{\varepsilon}=\lambda_{2}\left(A_{\varepsilon}-a_{2}\right)$, we introduce the following function

$$
U_{\varepsilon}(X)=\lambda_{2}^{\frac{2-n}{2}} u_{\varepsilon}\left(a_{2}+\lambda_{2}^{-1} X\right) .
$$

As in Lemma 2.3, we can prove $\lambda_{2} d\left(a_{2}, \partial A_{\varepsilon}\right) \rightarrow+\infty$. It is easy to see that $U_{\varepsilon}$ satisfies

$$
U_{\varepsilon} \leqslant 1, \quad \text { in } B\left(0,(1 / 2) \lambda_{2}\left|a_{1}-a_{2}\right|\right) .
$$

Thus, there exists $b \in \mathbb{R}^{n}$ and $\lambda>0$ such that $U_{\varepsilon} \rightarrow \delta_{(b, \lambda)}$ in $C_{\text {loc }}^{1}\left(\mathbb{R}^{n}\right)$. Therefore we have also found a second point of blow up $\bar{a}_{2}$ of $u_{\varepsilon}$ with the concentration $\bar{\lambda}_{2}$ in this case $\left(\bar{a}_{2}=a_{2}+b / \lambda_{2}\right.$ and $\left.\bar{\lambda}_{2}=\lambda \lambda_{2}\right)$.

Now, we study the second case, that is $h_{\varepsilon}$ remains bounded and $\int_{B\left(a_{1}, 2 \varepsilon\right)} \mid u_{\varepsilon}^{1} \frac{2 n}{n-2}^{\frac{2 n}{n}} \nrightarrow 0$ as $\varepsilon \rightarrow 0$. We introduce the following function defined on $F_{\varepsilon}=\varepsilon^{-1}\left(A_{\varepsilon}-a_{1}\right)$ by

$$
W_{\varepsilon}(X)=\varepsilon^{\frac{n-2}{2}} u_{\varepsilon}^{1}\left(a_{1}+\varepsilon X\right) .
$$

Observe that $F_{\varepsilon}$ "converges" to a strip of $\mathbb{R}^{n}$ when $\varepsilon \rightarrow 0$. We notice that $W_{\varepsilon}$ satisfies

$$
\begin{cases}-\Delta W_{\varepsilon}=\left|W_{\varepsilon}\right|^{\frac{4}{n-2}} W_{\varepsilon}+f_{\varepsilon} & \text { in } F_{\varepsilon}, \\ W_{\varepsilon}=0 & \text { on } \partial F_{\varepsilon},\end{cases}
$$

with $\left|f_{\varepsilon}\right|_{H^{-1}\left(F_{\varepsilon}\right)} \rightarrow 0$ as $\varepsilon \rightarrow 0$. We also have

$$
\int_{B(0,2) \cap F_{\varepsilon}}\left|W_{\varepsilon}\right|^{\frac{2 n}{n-2}}=\int_{B\left(a_{1}, 2 \varepsilon\right) \cap A_{\varepsilon}}\left|u_{\varepsilon}^{1}\right|^{\frac{2 n}{n-2}} \nrightarrow 0, \quad \text { as } \varepsilon \rightarrow 0
$$

and

$$
\int_{F_{\varepsilon}}\left|\nabla W_{\varepsilon}\right|^{2}=\int_{A_{\varepsilon}}\left|\nabla u_{\varepsilon}^{1}\right|^{2} \leqslant C
$$

It is easy to check that there exists some fixed domain $F \subset B(0,2) \cap F_{\varepsilon}$ such that $\left|W_{\varepsilon}\right|^{\frac{2 n}{n-2}} \rightarrow 0$ almost everywhere and $\left|W_{\varepsilon}\right|^{\frac{2 n}{n-2}} \nrightarrow 0$ in $L^{1}(F)$. From Dunford-Pettis Lemma [5], we have

$$
\exists \delta_{0}>0, \exists \alpha_{\varepsilon}>0, \alpha_{\varepsilon} \rightarrow 0, \exists b_{\varepsilon} \in F \text { s.t. } \int_{B\left(b_{\varepsilon}, \alpha_{\varepsilon}\right) \cap F_{\varepsilon}}\left|W_{\varepsilon}\right|^{\frac{2 n}{n-2}} \geqslant \delta_{0} .
$$


We can choose $b_{\varepsilon}$ and $\alpha_{\varepsilon}$ such that $\alpha_{\varepsilon}$ is minimum and $\int_{B\left(b_{\varepsilon}, \alpha_{\varepsilon}\right) \cap F_{\varepsilon}}\left|W_{\varepsilon}\right|^{\frac{2 n}{n-2}}=\delta_{0}$.

LeMma 2.5. - Let $\left(\alpha_{\varepsilon}, b_{\varepsilon}\right)$ be defined by (2.7) and let $\bar{\lambda}_{2}=\left(\varepsilon \alpha_{\varepsilon}\right)^{-1}$, and $\bar{a}_{2}=$ $a_{1}+\varepsilon b_{\varepsilon}$. Then we have

$$
\frac{\lambda_{1}}{\bar{\lambda}_{2}} \rightarrow+\infty \quad \text { or } \quad \frac{\bar{\lambda}_{2}}{\lambda_{1}} \rightarrow+\infty \quad \text { or } \quad \lambda_{1} \bar{\lambda}_{2}\left|a_{1}-\bar{a}_{2}\right|^{2} \rightarrow+\infty \quad \text { when } \varepsilon \rightarrow 0
$$

where $\lambda_{1}=M_{\varepsilon}^{\frac{2}{n-2}}$.

Proof. - We argue by contradiction. Let us suppose that $\lambda_{1} / \bar{\lambda}_{2}, \bar{\lambda}_{2} / \lambda_{1}$ and $\lambda_{1} \bar{\lambda}_{2} \mid a_{1}-$ $\left.\bar{a}_{2}\right|^{2}$ are bounded when $\varepsilon \rightarrow 0$.

For $X \in \widetilde{A}_{\varepsilon}:=\lambda_{1}\left(A_{\varepsilon}-a_{1}\right)$, we introduce $\omega_{\varepsilon}$ defined by

$$
\omega_{\varepsilon}(X)=M_{\varepsilon}^{-1} u_{\varepsilon}^{1}\left(a_{1}+\lambda_{1}^{-1} X\right) .
$$

Observe that, on the one hand

$$
\begin{aligned}
\int \omega_{B\left(\lambda_{1}\left(\bar{a}_{2}-a_{1}\right), \lambda_{1} / \bar{\lambda}_{2}\right) \cap \widetilde{A}_{\varepsilon}}\left|\omega_{\varepsilon}(X)\right|^{\frac{2 n}{n-2}} \mathrm{~d} X & =\int_{B\left(\bar{a}_{2}, 1 / \bar{\lambda}_{2}\right) \cap A_{\varepsilon}}\left|u_{\varepsilon}^{1}(x)\right|^{\frac{2 n}{n-2}} \mathrm{~d} x \\
& =\int_{B\left(b_{\varepsilon}, \alpha_{\varepsilon}\right) \cap F_{\varepsilon}}\left|W_{\varepsilon}(X)\right|^{\frac{2 n}{n-2}} \mathrm{~d} X=\delta_{0}>0 .
\end{aligned}
$$

On the other hand, since $\lambda_{1}\left|\bar{a}_{2}-a_{1}\right|$ and $\lambda_{1} / \bar{\lambda}_{2}$ are bounded, we have

$$
\exists R>0 \text { such that } B\left(\lambda_{1}\left(\bar{a}_{2}-a_{1}\right), \lambda_{1} / \bar{\lambda}_{2}\right) \subset B(0, R) .
$$

Thus

$$
\begin{aligned}
& \quad \int_{B\left(\lambda_{1}\left(\bar{a}_{2}-a_{1}\right), \lambda_{1} / \bar{\lambda}_{2}\right) \cap \widetilde{A}_{\varepsilon}}\left|\omega_{\varepsilon}(X)\right|^{\frac{2 n}{n-2}} \mathrm{~d} X \\
& \leqslant \int_{B(0, R) \cap \widetilde{A}_{\varepsilon}}\left|\omega_{\varepsilon}(X)\right|^{\frac{2 n}{n-2}} \mathrm{~d} X \\
& =\int_{B(0, R) \cap \widetilde{A}_{\varepsilon}}\left|M_{\varepsilon}^{-1} u_{\varepsilon}\left(a_{1}+\lambda_{1}^{-1} X\right)-M_{\varepsilon}^{-1} P_{\varepsilon} \delta_{\left(a_{1}, \lambda_{1}\right)}\left(a_{1}+\lambda_{1}^{-1} X\right)\right|^{\frac{2 n}{n-2}} \mathrm{~d} X \\
& \leqslant c \int_{B(0, R)}\left|v_{\varepsilon}-\delta_{\left(0, \alpha_{n}\right)}(X)\right|^{\frac{2 n}{n-2}}+c \int_{A_{\varepsilon}}\left|\delta_{\varepsilon}(x)-P_{\varepsilon} \delta(x)\right|^{\frac{2 n}{n-2}} .
\end{aligned}
$$

Thus

$$
\int_{B\left(\lambda_{1}\left(\bar{a}_{2}-a_{1}\right), \lambda_{1} \bar{\lambda}_{2}\right) \cap \widetilde{A}_{\varepsilon}}\left|W_{\varepsilon}(X)\right|^{\frac{2 n}{n-2}} \mathrm{~d} X \rightarrow 0 \quad \text { when } \varepsilon \rightarrow 0
$$

which yields a contradiction and our lemma follows. 
Now we set $\widetilde{\widetilde{A}}_{\varepsilon}=\bar{\lambda}_{2}\left(A_{\varepsilon}-\bar{a}_{2}\right)$ and we introduce the function $V_{\varepsilon}$ defined by

$$
V_{\varepsilon}(X)=\bar{\lambda}_{2}^{\frac{(2-n)}{2}} u_{\varepsilon}^{1}\left(\bar{a}_{2}+\bar{\lambda}_{2}^{-1} X\right) \text {. }
$$

Observe that

$$
\int_{B(0,1) \cap \widetilde{\widetilde{A}}_{\varepsilon}}\left|V_{\varepsilon}\right|^{\frac{2 n}{n-2}}=\int_{B\left(\bar{a}_{2}, 1 / \bar{\lambda}_{2}\right) \cap A_{\varepsilon}}\left|u_{\varepsilon}^{1}\right|^{\frac{2 n}{n-2}}=\delta_{0}>0
$$

and we also have

$$
\int\left|\nabla V_{\varepsilon}\right|^{2} \leqslant C, \quad \int\left|V_{\varepsilon}\right|^{\frac{2 n}{n-2}} \leqslant C .
$$

It is easy to see that there exists some functions $\mathrm{V}$ such that (after passing to a subsequence), $V_{\varepsilon} \rightarrow V$ in $H_{\text {loc }}^{1}(\Omega)$ and $\mathrm{V}$ satisfies

$$
\left\{\begin{array}{l}
-\Delta V=|V|^{\frac{4}{n-2}} V \quad \text { in } \Omega, \quad V=0 \quad \text { on } \partial \Omega, \\
\int_{\Omega}|\nabla V|^{2} \leqslant C, \quad \int_{\Omega}|V|^{\frac{2 n}{n-2}} \leqslant C,
\end{array}\right.
$$

where $\Omega$ is a half space or a strip or a $\mathbb{R}^{n}$.

From (2.9), it is easy to see that $V \neq 0$.

LEMMA 2.6. - Let $V$ be defined by (2.10). Then, we have $V \geqslant 0$.

Proof. - We have

$$
\begin{aligned}
V_{\varepsilon}(X)= & \bar{\lambda}_{2}^{\frac{(2-n)}{2}} u_{\varepsilon}\left(\bar{a}_{2}+\bar{\lambda}_{2}^{-1} X\right)-\bar{\lambda}_{2}^{\frac{(2-n)}{2}} \delta_{\left(a_{1}, \lambda_{1}\right)}\left(\bar{a}_{2}+\bar{\lambda}_{2}^{-1} X\right) \\
& +\bar{\lambda}_{2}^{\frac{(2-n)}{2}}\left(\delta_{\left(a_{1}, \lambda_{1}\right)}\left(\bar{a}_{2}+\bar{\lambda}_{2}^{-1} X\right)-P_{\varepsilon} \delta_{\left(a_{1}, \lambda_{1}\right)}\left(\bar{a}_{2}+\bar{\lambda}_{2}^{-1} X\right)\right) .
\end{aligned}
$$

Thus, it is sufficient to prove that

$$
\bar{\lambda}_{2}^{\frac{(2-n)}{2}} \delta_{\left(a_{1}, \lambda_{1}\right)}\left(\bar{a}_{2}+\bar{\lambda}_{2}^{-1} X\right) \rightarrow 0 \quad \text { in } H_{\mathrm{loc}}^{1}\left(\mathbb{R}^{n}\right)
$$

Observe that

$$
\begin{aligned}
I_{\varepsilon} & :=\int_{B(0, R)}\left(\bar{\lambda}_{2}^{\frac{(2-n)}{2}} \frac{\lambda_{1}^{\frac{n-2}{2}}}{\left(1+\lambda_{1}^{2}\left|\bar{a}_{2}+\bar{\lambda}_{2}^{-1} X-a_{1}\right|^{2}\right)^{\frac{n-2}{2}}}\right)^{\frac{n+2}{n-2}} \mathrm{~d} X \\
& =\left(\frac{\lambda_{1}}{\bar{\lambda}_{2}}\right)^{\frac{n+2}{2}} \int_{B(0, R)} \frac{\mathrm{d} X}{\left(1+\left(\lambda_{1} / \bar{\lambda}_{2}\right)^{2}\left|X-\bar{\lambda}_{2}\left(a_{1}-\bar{a}_{2}\right)\right|^{2}\right)^{\frac{n+2}{2}}} .
\end{aligned}
$$

If $\lambda_{1} / \bar{\lambda}_{2} \rightarrow 0$ it is clear that $I_{\varepsilon} \rightarrow 0$ when $\varepsilon \rightarrow 0$. If $\lambda_{1} \bar{\lambda}_{2} \rightarrow+\infty$, let $y=\left(\lambda_{1} / \bar{\lambda}_{2}\right) X$. Thus

$$
I_{\varepsilon} \leqslant\left(\frac{\bar{\lambda}_{2}}{\lambda_{1}}\right)^{\frac{n-2}{2}} \int_{\mathbb{R}^{n}}\left(\frac{1}{1+\left|y-\lambda_{1}\left(a_{1}-\bar{a}_{2}\right)\right|^{2}}\right)^{\frac{n+2}{2}} \mathrm{~d} y \rightarrow 0 \quad \text { when } \varepsilon \rightarrow 0
$$


Lastly if $\lambda_{1} / \bar{\lambda}_{2} \not \rightarrow+\infty$ and $\bar{\lambda}_{2} / \lambda_{1} \not \nrightarrow+\infty$, then, by Lemma 2.5 , we have

$$
\lambda_{1} \bar{\lambda}_{2}\left|a_{1}-\bar{a}_{2}\right|^{2} \rightarrow+\infty, \quad \text { when } \varepsilon \rightarrow 0 .
$$

Observe that for $X \in B(0, R)$, we have

$$
\left|X-\bar{\lambda}_{2}\left(a_{1}-\bar{a}_{2}\right)\right| \geqslant\left|\bar{\lambda}_{2}\left(a_{1}-\bar{a}_{2}\right)\right|-|X| \geqslant c\left|\bar{\lambda}_{2}\left(a_{1}-\bar{a}_{2}\right)\right| .
$$

Therefore

$$
\begin{aligned}
I_{\varepsilon} & \leqslant\left(\frac{\lambda_{1}}{\bar{\lambda}_{2}}\right)^{\frac{n+2}{2}} \int_{B(0, R)} \frac{1}{\left(c\left(\frac{\lambda_{1}}{\bar{\lambda}_{2}}\right)^{2}\left|\bar{\lambda}_{2}\left(a_{1}-\bar{a}_{2}\right)\right|^{2}\right)^{\frac{n+2}{2}}} \\
& \leqslant \frac{1}{\left(\lambda_{1} \bar{\lambda}_{2}\left|a_{1}-\bar{a}_{2}\right|^{2}\right)^{\frac{n+2}{2}}} C(R) \rightarrow 0, \quad \text { when } \varepsilon \rightarrow 0 .
\end{aligned}
$$

Then, our lemma follows.

Now, from Theorem III 1.3 [15], we derive $\Omega=\mathbb{R}^{n}$. Thus, using (2.10) and Lemma 2.6, we also obtain a second point of blow up of $u_{\varepsilon}$ in this case. Thus in all cases we have built a second point $a_{2, \varepsilon}$ of blow up of $u_{\varepsilon}$ with the concentration $\lambda_{2, \varepsilon}$ such that $\lambda_{2, \varepsilon} \rightarrow+\infty$ and $\lambda_{2, \varepsilon} d\left(a_{2, \varepsilon}, \partial A_{\varepsilon}\right) \rightarrow+\infty$ as $\varepsilon \rightarrow 0$. It is clear that we can proceed by inductions. Thus, we obtain a sequence $\left(u_{\varepsilon}^{k}\right)_{k}$ such that

$$
\int_{A_{\varepsilon}}\left|\nabla u_{\varepsilon}^{k}\right|^{2}=\int_{A_{\varepsilon}}\left|\nabla u_{\varepsilon}^{k-1}\right|^{2}-S_{n}+\mathrm{o}(1)=\int_{A_{\varepsilon}}\left|\nabla u_{\varepsilon}\right|^{2}-k S_{n}+\mathrm{o}(1) .
$$

Thus

$$
0 \leqslant \int_{A_{\varepsilon}}\left|\nabla u_{\varepsilon}^{k}\right|^{2}=\int_{A_{\varepsilon}}\left|\nabla u_{\varepsilon}\right|^{2}-k S_{n}+\mathrm{o}(1) \leqslant C-k S_{n}+\mathrm{o}(1) .
$$

Since the later term in (2.13) will be negative for large k, the induction will terminate after some index $p \in \mathbb{N}^{*}$. Moreover, for this index, we have

$$
\left|\nabla\left(u_{\varepsilon}-\sum_{i=1}^{p} P_{\varepsilon} \delta_{\left(a_{i, \varepsilon}, \lambda_{i, \varepsilon}\right)}\right)\right|_{L^{2}\left(A_{\varepsilon}\right)} \rightarrow 0, \quad \lambda_{i, \varepsilon} \rightarrow+\infty,
$$

$\lambda_{i, \varepsilon} d\left(a_{i, \varepsilon}, \partial A_{\varepsilon}\right) \rightarrow+\infty$,

$$
\forall i \neq j, \quad \varepsilon_{i j}=\left(\frac{\lambda_{i, \varepsilon}}{\lambda_{j, \varepsilon}}+\frac{\lambda_{j, \varepsilon}}{\lambda_{i, \varepsilon}}+\lambda_{i, \varepsilon} \lambda_{j, \varepsilon}\left|a_{i, \varepsilon}-a_{j, \varepsilon}\right|^{2}\right)^{-\frac{n-2}{2}} \rightarrow 0
$$

as desired in the first part of Theorem 1.3. 


\section{Proof of the second part of Theorem 1.3}

Let, for $p \in \mathbb{N}^{*}$ and $\eta>0$ given

$$
\begin{aligned}
V_{\varepsilon}(p, \eta)= & \left\{u \in \Sigma^{+}\left(A_{\varepsilon}\right) \text { s.t. } \exists x_{1}, \ldots, x_{p} \in A_{\varepsilon}, \exists \lambda_{1}, \ldots, \lambda_{p}>0\right. \text { with } \\
& \left|u-C(p) \sum_{i=1}^{p} P_{\varepsilon} \delta_{\left(x_{i}, \lambda_{i}\right)}\right|_{H_{0}^{1}\left(A_{\varepsilon}\right)}<\eta, \forall i \lambda_{i}>\frac{1}{\eta}, \lambda_{i} d\left(x_{i}, \partial A_{\varepsilon}\right) \geqslant \frac{1}{\eta}, \\
& \left.\forall i \neq j \quad \varepsilon_{i j}=\left(\frac{\lambda_{i}}{\lambda_{j}}+\frac{\lambda_{j}}{\lambda_{i}}+\lambda_{i} \lambda_{j}\left|x_{i}-x_{j}\right|^{2}\right)^{-\frac{n-2}{2}}<\eta\right\}
\end{aligned}
$$

where $\Sigma^{+}\left(A_{\varepsilon}\right)=\left\{\left.u \in H_{0}^{1}\left(A_{\varepsilon}\right)|u \geqslant 0| u\right|_{,H_{0}^{1}\left(A_{\varepsilon}\right)}=1\right\}$.

If a function $u$ belongs to $V_{\varepsilon}(p, \eta)$, then, for $\eta>0$ small enough, the minimization problem

$$
\min _{\alpha_{i}, \lambda_{i}>0, x_{i} \in A_{\varepsilon}}\left|u-\sum_{i=1}^{p} \alpha_{i} P_{\varepsilon} \delta_{\left(x_{i}, \lambda_{i}\right)}\right|_{H_{0}^{1}\left(A_{\varepsilon}\right)}
$$

has a unique solution, up to permutation (see Lemma A.2 in [3]).

Therefore, for $\varepsilon>0$ sufficiently small, Section 2 implies that $u_{\varepsilon}$ (solution of $P_{\varepsilon}$ ) can be uniquely writen as

$$
\tilde{u}_{\varepsilon}=\frac{u_{\varepsilon}}{\left|u_{\varepsilon}\right|_{H_{0}^{1}}}=\sum \alpha_{i, \varepsilon} P_{\varepsilon} \delta_{\left(a_{i, \varepsilon}, \lambda_{i, \varepsilon}\right)}+v_{\varepsilon}
$$

where $v_{\varepsilon}$ satisfies the following conditions:

$$
\begin{aligned}
\left(V_{0}\right) \quad\left(v_{\varepsilon}, P_{\varepsilon} \delta_{\left(a_{i, \varepsilon}, \lambda_{i, \varepsilon}\right)}\right)_{H_{0}^{1}} & =\left(v_{\varepsilon}, \frac{\partial P_{\varepsilon} \delta_{\left(a_{i, \varepsilon}, \lambda_{i, \varepsilon}\right)}}{\partial \lambda_{i}}\right)_{H_{0}^{1}} \\
& =\left(v_{\varepsilon}, \frac{\partial P_{\varepsilon} \delta_{\left(a_{i, \varepsilon}, \lambda_{i, \varepsilon}\right)}}{\partial a_{i}}\right)_{H_{0}^{1}}=0
\end{aligned}
$$

and $\alpha_{i, \varepsilon}$ satisfies:

$$
\left(J\left(u_{\varepsilon}\right)\right)^{\frac{n}{n-2}} \alpha_{j}^{\frac{4}{n-2}}=1+o(1), \quad \forall j .
$$

In order to simplify the notations, in the remainder, we write $\alpha_{i}, a_{i}, \lambda_{i}, \delta_{i}$ and $P \delta_{i}$ instead of $\alpha_{i, \varepsilon}, a_{i, \varepsilon}, \lambda_{i, \varepsilon}, \delta_{a_{i, \varepsilon}, \lambda_{i, \varepsilon}}$ and $P \delta_{a_{i, \varepsilon}, \lambda_{i, \varepsilon}}$ respectively and we also write $u_{\varepsilon}$ instead of $\tilde{u}_{\varepsilon}$.

As usual in this type of problems, we first deal with the $v_{\varepsilon}$-part of $u_{\varepsilon}$.

PROPOSITION 3.1. - Let $v_{\varepsilon}$ be defined by (3.2). Then, we have the following estimate

$$
\left|v_{\varepsilon}\right|_{H_{0}^{1}\left(A_{\varepsilon}\right)} \leqslant C \begin{cases}\sum_{i} \frac{1}{\left(\lambda_{i} d_{i}\right)^{n-2}}+\sum \varepsilon_{i j}\left(\log \left(\varepsilon_{i j}^{-1}\right)\right)^{\frac{n-2}{n}} & \text { if } n<6, \\ \sum_{i} \frac{1}{\left(\lambda_{i} d_{i}\right)^{\frac{n+2}{2}}}+\sum \varepsilon_{i j}^{\frac{n+2}{2(n-2)}}\left(\log \left(\varepsilon_{i j}^{-1}\right)\right)^{\frac{n+2}{2 n}} & \text { if } n \geqslant 6 .\end{cases}
$$


Proof. - From (3.2), we derive

$$
\begin{aligned}
-\Delta v_{\varepsilon}= & -\Delta u_{\varepsilon}+\Delta\left(\sum \alpha_{i} P \delta_{i}\right) \\
= & \left(J\left(u_{\varepsilon}\right)\right)^{\frac{n}{n-2}}\left(\sum \alpha_{i} P \delta_{i}+v_{\varepsilon}\right)^{\frac{n+2}{n-2}}-\sum \alpha_{i} \delta_{i}^{\frac{n+2}{n-2}} \\
= & \left(J\left(u_{\varepsilon}\right)\right)^{\frac{n}{n-2}}\left[\left(\sum \alpha_{i} P \delta_{i}\right)^{\frac{n+2}{n-2}}+\frac{n+2}{n-2}\left(\sum \alpha_{i} P \delta_{i}\right)^{\frac{4}{n-2}} v_{\varepsilon}+\mathrm{O}\left(\left|v_{\varepsilon}\right|^{\frac{n+2}{n-2}}\right)\right. \\
& \left.+\mathrm{O}\left(\operatorname{Sup}\left(\sum \alpha_{i} P \delta_{i}, v_{\varepsilon}\right)^{\frac{4}{n-2}-1}\left|v_{\varepsilon}\right|^{2}\right)\right]-\sum \alpha_{i} \delta_{i}^{\frac{n+2}{n-2}} .
\end{aligned}
$$

Thus, since $J\left(u_{\varepsilon}\right)$ is bounded,

$$
\int_{A_{\varepsilon}}\left|\nabla v_{\varepsilon}\right|^{2}=\left(J\left(u_{\varepsilon}\right)\right)^{\frac{n}{n-2}}\left[\langle f, v\rangle+\frac{n+2}{n-2} \int_{A_{\varepsilon}}\left(\sum \alpha_{i} P \delta_{i}\right)^{\frac{4}{n-2}} v_{\varepsilon}^{2}\right]+\mathrm{O}\left(\left|v_{\varepsilon}\right|_{H_{0}^{1}}^{\inf (3,2 n /(n-2)}\right)
$$

where

$$
\langle f, v\rangle=\int_{A_{\varepsilon}}\left(\sum \alpha_{i} P \delta_{i}\right)^{\frac{n+2}{n-2}} v
$$

Then

$$
Q\left(v_{\varepsilon}, v_{\varepsilon}\right)=\left(J\left(u_{\varepsilon}\right)\right)^{\frac{n}{n-2}}\left\langle f, v_{\varepsilon}\right\rangle+\mathrm{O}\left(\left|v_{\varepsilon}\right|_{H_{0}^{1}}^{\inf (3,2 n /(n-2))}\right),
$$

where

$$
Q\left(v_{\varepsilon}, v_{\varepsilon}\right)=\int_{A_{\varepsilon}}\left|\nabla v_{\varepsilon}\right|^{2}-\frac{n+2}{n-2}\left(J\left(u_{\varepsilon}\right)\right)^{\frac{n}{n-2}} \int_{A_{\varepsilon}}\left(\sum \alpha_{i} P \delta_{i}\right)^{\frac{4}{n-2}} v_{\varepsilon}^{2} .
$$

Observe that, since $J\left(u_{\varepsilon}\right)^{\frac{n}{n-2}} \alpha_{i}^{\frac{4}{n-2}}=1+\mathrm{o}(1)$, then $Q(v, v)$ is close to

$$
\int_{A_{\varepsilon}}|\nabla v|^{2}-\frac{n+2}{n-2} \sum_{i} \int_{A_{\varepsilon}} P \delta_{i}^{\frac{4}{n-2}} v^{2}
$$

and therefore $Q$ is a positive definite quadratic form on $v$ (see [2]). Thus

Hence

$$
\begin{aligned}
\alpha_{0}\left|v_{\varepsilon}\right|_{H_{0}^{1}}^{2} & \leqslant Q\left(v_{\varepsilon}, v_{\varepsilon}\right)+\mathrm{O}\left(\left|v_{\varepsilon}\right|_{H_{0}^{1}}^{\inf (3,2 n / n-2)}\right) \\
& =\left(J\left(u_{\varepsilon}\right)\right)^{\frac{n}{n-2}}\left\langle f, v_{\varepsilon}\right\rangle \\
& \leqslant C|f|\left|v_{\varepsilon}\right|_{H_{0}^{1}} .
\end{aligned}
$$

$$
\left|v_{\varepsilon}\right|_{H_{0}^{1}} \leqslant C^{\prime}|f|
$$

Now we estimate $|f|$. We have

$$
\left\langle f, v_{\varepsilon}\right\rangle=\sum \alpha_{i}^{\frac{n+2}{n-2}} \int_{A_{\varepsilon}} P \delta_{i}^{\frac{n+2}{n-2}} v_{\varepsilon}+\mathrm{O}\left(\sum \int_{P \delta_{j} \leqslant P \delta_{i}} P \delta_{i}^{\frac{4}{n-2}} P \delta_{j}\left|v_{\varepsilon}\right|\right) .
$$


Observe that

$$
\begin{aligned}
\int_{A_{\varepsilon}} P \delta_{i}^{\frac{n+2}{n-2}} v_{\varepsilon} & =\int_{A_{\varepsilon}} v_{\varepsilon} \delta_{i}^{\frac{n+2}{n-2}}+\mathrm{O}\left(\int_{A_{\varepsilon}} \delta_{i}^{\frac{4}{n-2}}\left(\delta_{i}-P \delta_{i}\right)\left|v_{\varepsilon}\right|\right) \\
& =\mathrm{O}\left(\int_{B\left(a_{i}, d_{i}\right)} \delta_{i}^{\frac{4}{n-2}}\left(\delta_{i}-P \delta_{i}\right)\left|v_{\varepsilon}\right|\right)+\mathrm{O}\left(\int_{\mathbb{R}^{n} \backslash B\left(a_{i}, d_{i}\right)} \delta_{i}^{\frac{n+2}{n-2}}\left|v_{\varepsilon}\right|\right)
\end{aligned}
$$

where $d_{i}=d\left(a_{i}, \partial A_{\varepsilon}\right)$. Thus

$$
\begin{aligned}
\int_{A_{\varepsilon}} P \delta_{i}^{\frac{n+2}{n-2}} v_{\varepsilon}= & \mathrm{O}\left(| v _ { \varepsilon } | _ { H _ { 0 } ^ { 1 } } \left[\left|\delta_{i}-P \delta_{i}\right|_{L^{\infty}}\left(\int_{B\left(a_{i}, d_{i}\right)} \delta_{i}^{\frac{8 n}{n^{2}-4}}\right)^{\frac{n+2}{2 n}}\right.\right. \\
& \left.\left.+\left(\int_{B^{c}\left(a_{i}, d_{i}\right)} \delta_{i}^{\frac{2 n}{n-2}}\right)^{\frac{n+2}{2 n}}\right]\right) .
\end{aligned}
$$

Notice that

$$
\int_{\mathbb{R}^{n} \backslash B\left(a_{i}, d_{i}\right)} \delta_{i}^{\frac{2 n}{n-2}}=\mathrm{O}\left(\frac{1}{\left(\lambda_{i} d_{i}\right)^{n}}\right)
$$

and

$$
\begin{aligned}
& \left(\int_{B\left(a_{i}, d_{i}\right)} \delta_{i}^{\frac{8 n}{n^{2}-4}}\right)^{\frac{n+2}{2 n}} \\
& \quad=\mathrm{O}\left(\frac{d_{i}^{\frac{n-6}{2}}}{\lambda_{i}^{2}}(\text { if } n>6)+\frac{\log \left(\lambda_{i} d_{i}\right)}{\lambda_{i}^{2}}(\text { if } n=6)+\frac{1}{\lambda_{i}^{\frac{n-2}{2}}}(\text { if } n<6)\right)
\end{aligned}
$$

Therefore

$$
\begin{aligned}
& \int_{A_{\varepsilon}} P \delta_{i}^{\frac{n+2}{n-2}} v_{\varepsilon} \\
& \quad=\mathrm{O}\left(\left|v_{\varepsilon}\right|_{H_{0}^{1}}\left[\frac{1}{\left(\lambda_{i} d_{i}\right)^{\frac{n+2}{2}}}+(\text { if } n=6) \frac{\log \left(\lambda_{i} d_{i}\right)}{\left(\lambda_{i} d_{i}\right)^{4}}+(\text { if } n<5) \frac{1}{\left(\lambda_{i} d_{i}\right)^{n-2}}\right]\right) .
\end{aligned}
$$

We also have

$$
\int_{P \delta_{j} \leqslant P \delta_{i}} P \delta_{i}^{\frac{4}{n-2}} P \delta_{j}\left|v_{\varepsilon}\right| \leqslant\left|v_{\varepsilon}\right|\left[\int_{P \delta_{j} \leqslant P \delta_{i}}\left(\left(P \delta_{i}\right)^{\frac{4}{n-2}} P \delta_{j}\right)^{\frac{2 n}{n+2}}\right]^{\frac{n+2}{2 n}} .
$$

If $n \geqslant 6$, we have $\frac{2 n}{n+2} \geqslant \frac{n}{n-2}$ and thus

$$
\int_{P \delta_{j} \leqslant P \delta_{i}}\left(P \delta_{i}^{\frac{4}{n-2}} P \delta_{j}\right)^{\frac{2 n}{n+2}} \leqslant \int_{P \delta_{j} \leqslant P \delta_{i}}\left(P \delta_{i} P \delta_{j}\right)^{\frac{n}{n-2}}=\mathrm{O}\left(\varepsilon_{i j}^{\frac{n}{n-2}} \log \left(\varepsilon_{i j}^{-1}\right)\right) .
$$

If $n \leqslant 5$, we have $1<\frac{4}{n-2}$, thus 


$$
\int_{P \delta_{j} \leqslant P \delta_{i}}\left(P \delta_{i}^{\frac{4}{n-2}} P \delta_{j}\right)^{\frac{2 n}{n+2}} \leqslant c\left(\int_{P \delta_{j} \leqslant P \delta_{i}}\left(\delta_{i} \delta_{j}\right)^{\frac{n}{n-2}}\right)^{\frac{2(n-2)}{n+2}} \leqslant c \varepsilon_{i j}^{\frac{2 n}{n+2}}\left(\log \left(\varepsilon_{i j}^{-1}\right)\right)^{\frac{2(n-2)}{n+2}} .
$$

Using (3.6), (3.7), (3.8) and (3.9) we conclude that

$$
|f| \leqslant \begin{cases}\sum \frac{1}{\left(\lambda_{i} d_{i}\right)^{n-2}}+\sum \varepsilon_{i j}\left(\log \left(\varepsilon_{i j}^{-1}\right)\right)^{\frac{(n-2)}{n}} & \text { if } n<6, \\ \sum \frac{1}{\left(\lambda_{i} d_{i}\right)^{\frac{n+2}{2}}}+\sum \varepsilon_{i j}^{\frac{(n+2)}{2(n-2)}}\left(\log \left(\varepsilon_{i j}^{-1}\right)\right)^{\frac{(n+2)}{2 n}} & \text { if } n \geqslant 6\end{cases}
$$

and Proposition 3.1 follows.

PROPOSITION 3.2. - For $n \geqslant 4$, we have the following expansion

$$
\begin{aligned}
\left(\nabla J\left(u_{\varepsilon}\right), \lambda_{i} \frac{\partial P \delta_{i}}{\partial \lambda_{i}}\right)_{H_{0}^{1}\left(A_{\varepsilon}\right)}= & 2 J\left(u_{\varepsilon}\right) c_{1}\left[-\frac{(n-2)}{2} \alpha_{i} \frac{H_{\varepsilon}\left(a_{i}, a_{i}\right)}{\lambda_{i}^{n-2}}(1+\mathrm{o}(1))\right. \\
& \left.-\sum \alpha_{j}\left(\lambda_{i} \frac{\partial \varepsilon_{i j}}{\partial \lambda_{i}}+\frac{(n-2)}{2} \frac{H_{\varepsilon}\left(a_{i}, a_{j}\right)}{\left(\lambda_{i} \lambda_{j}\right)^{\frac{n-2}{2}}}\right)(1+\mathrm{o}(1))+R\right],
\end{aligned}
$$

where

$$
R=\mathrm{O}\left(\sum_{k} \frac{\log \left(\lambda_{k} d_{k}\right)}{\left(\lambda_{k} d_{k}\right)^{n}}+\sum_{i \neq j} \varepsilon_{i j}^{\frac{n}{n-2}} \log \left(\varepsilon_{i j}^{-1}\right)\right)
$$

Proof. - We have

$$
\nabla J\left(u_{\varepsilon}\right)=2 J\left(u_{\varepsilon}\right)\left[u_{\varepsilon}+J\left(u_{\varepsilon}\right)^{\frac{n}{n-2}} \Delta^{-1}\left(u_{\varepsilon}^{\frac{n+2}{n-2}}\right)\right] .
$$

Thus

$$
\begin{aligned}
\left(\nabla J\left(u_{\varepsilon}\right), \lambda_{i} \frac{\partial P \delta_{i}}{\partial \lambda_{i}}\right)= & 2 J\left(u_{\varepsilon}\right)\left[\sum \alpha_{j}\left(P \delta_{j}, \lambda_{i} \frac{\partial P \delta_{i}}{\partial \lambda_{i}}\right)\right. \\
& -J\left(u_{\varepsilon}\right)^{\frac{n}{n-2}}\left[\lambda_{i} \int\left(\sum \alpha_{j} P \delta_{j}\right)^{\frac{n+2}{n-2}} \frac{\partial P \delta_{i}}{\partial \lambda_{i}}\right. \\
& +\frac{n+2}{n-2} \int\left(\sum \alpha_{j} P \delta_{j}\right)^{\frac{4}{n-2}} v_{\varepsilon} \lambda_{i} \frac{\partial P \delta_{i}}{\partial \lambda_{i}} \\
& \left.\left.+\mathrm{O}\left(\left|v_{\varepsilon}\right|_{H_{0}^{1}}^{\inf (2,(n+2) /(n-2))}\right)\right]\right] .
\end{aligned}
$$

Notice that if $n \geqslant 4$, we have

$$
\begin{aligned}
\left(\sum \alpha_{j} P \delta_{j}\right)^{\frac{n+2}{n-2}}= & \sum\left(\alpha_{j} P \delta_{j}\right)^{\frac{n+2}{n-2}}+\frac{n+2}{n-2} \sum_{i \neq j}\left(\alpha_{i} P \delta_{i}\right)^{\frac{4}{n-2}} \alpha_{j} P \delta_{j} \\
& +\mathrm{O}\left(\sum_{i \neq j}\left(\alpha_{i} P \delta_{i}\right)^{\frac{2}{n-2}}\left(\alpha_{j} P \delta_{j}\right)^{\frac{n}{n-2}}\right) \\
& +\mathrm{O}\left(\sum_{k \notin\{i, j\}, i \neq j}\left(\alpha_{j} P \delta_{j}\right)^{\frac{4}{n-2}}\left(\alpha_{k} P \delta_{k}\right)\right)
\end{aligned}
$$


and

$$
\left(\sum \alpha_{j} P \delta_{j}\right)^{\frac{4}{n-2}}=\left(\alpha_{i} P \delta_{i}\right)^{\frac{4}{n-2}}+\mathrm{O}\left(\sum_{j \neq i} P \delta_{i}^{\frac{4}{n-2}-1} P \delta_{j}+P \delta_{j}^{\frac{4}{n-2}}\right) .
$$

Combining (3.12), (3.13) and (3.11) and using the fact that $\left|\lambda_{i} \partial P \delta_{i} / \partial \lambda_{i}\right| \leqslant c \delta_{i}$ and $P \delta_{k} \leqslant \delta_{k}$, we need to estimate the following integrals

$$
\begin{aligned}
& \int_{A_{\varepsilon}} \delta_{j} \delta_{i}^{\frac{4}{n-2}}\left|v_{\varepsilon}\right| \leqslant \int_{\delta_{j} \leqslant\left|v_{\varepsilon}\right|}+\int_{\delta_{i} \leqslant\left|v_{\varepsilon}\right|}+\int_{\left|v_{\varepsilon}\right| \leqslant \inf \left(\delta_{i}, \delta_{j}\right)} \\
& \leqslant \mathrm{O}\left(\left|v_{\varepsilon}\right|_{H_{0}^{1}}^{\frac{n+2}{n-2}}\right)+\mathrm{O}\left(\left|v_{\varepsilon}\right|_{H_{0}^{1}}^{2}\right)+\mathrm{O}\left(\int_{A_{\varepsilon}}\left(\delta_{j} \delta_{i}\right)^{\frac{n}{n-2}}\right) \\
& \int_{B\left(a_{i}, d_{i}\right)} P \delta_{i}^{\frac{4}{n-2}} v_{\varepsilon} \lambda_{i} \frac{\partial P \delta_{i}}{\partial \lambda_{i}} \\
& =-\int_{B_{i}} P \delta_{i}^{\frac{4}{n-2}} v_{\varepsilon} \lambda_{i} \frac{\partial\left(\delta_{i}-P \delta_{i}\right)}{\partial \lambda_{i}}+\int_{B_{i}} P \delta_{i}^{\frac{4}{n-2}} v_{\varepsilon} \lambda_{i} \frac{\partial \delta_{i}}{\partial \lambda_{i}} \\
& \leqslant \frac{\left|v_{\varepsilon}\right|_{H_{0}^{1}}}{\left(\lambda_{i} d_{i}^{2}\right)^{\frac{n-2}{2}}}\left(\int \delta^{\frac{8 n}{n^{2}-4}}\right)^{\frac{n+2}{2 n}}+\int_{B_{i}} \delta_{i}^{\frac{4}{n-2}} v_{\varepsilon} \lambda_{i} \frac{\partial \delta_{i}}{\partial \lambda_{i}}+\mathrm{O}\left(\int_{B_{i}} \delta_{i}^{\frac{4}{n-2}} v_{\varepsilon}\left(\delta_{i}-P \delta_{i}\right)\right) \\
& =\mathrm{O}\left(\left|v_{\varepsilon}\right|_{H_{0}^{1}}\right)\left(\frac{1}{\left(\lambda_{i} d_{i}\right)^{\frac{n+2}{2}}}+(\text { if } n=6) \frac{\log \left(\lambda_{i} d_{i}\right)}{\left(\lambda_{i} d_{i}\right)^{4}}+(\text { if } n \leqslant 5) \frac{1}{\left(\lambda_{i} d_{i}\right)^{n-2}}\right) \text {, } \\
& \int_{\mathbb{R}^{n} \backslash B\left(a_{i}, d_{i}\right)} P \delta_{i}^{\frac{4}{n-2}} v_{\varepsilon} \lambda_{i} \frac{\partial P \delta_{i}}{\partial \lambda_{i}}=\mathrm{O}\left(\left|v_{\varepsilon}\right|_{H_{0}^{1}} \frac{1}{\left(\lambda_{i} d_{i}\right)^{\frac{n+2}{2}}}\right) .
\end{aligned}
$$

Now, using Proposition 3.1, Lemmas A.5-A.9 in Appendix A, and the fact that

$$
J\left(u_{\varepsilon}\right)^{\frac{n}{n-2}} \alpha_{j}^{\frac{4}{n-2}}=1+o(1),
$$

Proposition 3.2 follows.

PROPOSITION 3.3. - For $n \geqslant 4$, we have the following expansion

$$
\begin{aligned}
\left(\nabla J\left(u_{\varepsilon}\right), \frac{1}{\lambda_{i}} \frac{\partial P \delta_{i}}{\partial a_{i}}\right)_{H_{0}^{1}}= & J(u) c_{1}\left[\frac{\alpha_{i}}{\lambda_{i}^{n-1}} \frac{\partial H_{\varepsilon}\left(a_{i}, a_{i}\right)}{\partial a_{i}}(1+\mathrm{o}(1))\right. \\
& -2 \sum \alpha_{j}\left(\frac{1}{\lambda_{i}} \frac{\partial \varepsilon_{i j}}{\partial a_{i}}-\frac{1}{\lambda_{i}\left(\lambda_{i} \lambda_{j}\right)^{\frac{n-2}{2}}} \frac{\partial H_{\varepsilon}\left(a_{i}, a_{j}\right)}{\partial a_{i}}\right)(1+\mathrm{o}(1)) \\
& \left.+\mathrm{O}\left(R+\sum \lambda_{j}\left|a_{i}-a_{j}\right| \varepsilon_{i j}^{\frac{n+1}{n-2}}\right)\right]
\end{aligned}
$$

where $R$ is defined in Proposition 3.2. 
Proof. - As in the proof of Proposition 3.2, we obtain (3.11) but with $\frac{1}{\lambda_{i}} \frac{\partial P \delta_{i}}{\partial a_{i}}$ instead of $\lambda_{i} \frac{\partial P \delta_{i}}{\partial \lambda_{i}}$ and using Lemmas A.10-A.14 in Appendix A our proposition follows.

Next we are going to give the proof of the second part of Theorem 1.3. From Proposition 3.2 we easily derive that $p \geqslant 2$. Now for $i \in\{1, \ldots, p\}$, we introduce the following condition

$$
2^{-p-1} \sum_{k \neq i} \varepsilon_{k i} \leqslant \sum_{j=1}^{p} H_{\varepsilon}\left(a_{i}, a_{j}\right)\left(\lambda_{i} \lambda_{j}\right)^{\frac{2-n}{2}} .
$$

We divide the set $\{1, \ldots, p\}$ into $T_{1} \cup T_{2}$ with

$$
\begin{aligned}
& T_{1}=\{i \mid \text { s.t. } i \text { satisfies (3.17) }\}, \\
& T_{2}=\{i \mid \text { s.t. } i \text { does not satisfy (3.17) }\} .
\end{aligned}
$$

In $T_{2}$ we order the $\lambda_{i}^{\prime} s: \lambda_{i_{1}} \leqslant \lambda_{i_{2}} \leqslant \cdots \leqslant \lambda_{i_{s}}$. We begin by proving the following lemma

LEMMA 3.4. - For $n \geqslant 4$, we have the following estimate

$$
\sum_{j \in T_{2}, j \neq i}\left(\varepsilon_{i j}+\left(\lambda_{i} d_{i}\right)^{2-n}\right)=R_{1}
$$

where $R_{1}=\mathrm{O}\left[\sum_{k \in T_{1}}\left(\frac{\log \lambda_{k} d_{k}}{\left(\lambda_{k} d_{k}\right)^{n}}+\sum_{r \neq k, r \in T_{1}} \varepsilon_{k r}^{\frac{n}{n-2}} \log \left(\varepsilon_{k r}^{-1}\right)\right)\right]$.

Proof. - Using Proposition 3.2, we derive

$$
\begin{aligned}
0= & \sum_{k=1}^{s} 2^{k} \alpha_{i_{k}}\left(\nabla J\left(u_{\varepsilon}\right), \lambda_{i_{k}} \frac{\partial P \delta_{i_{k}}}{\partial \lambda_{i_{k}}}\right) \\
= & 2 J\left(u_{\varepsilon}\right) c_{1} \sum_{k=1}^{s}\left[-\sum_{j \neq i_{k}} 2^{k} \alpha_{j} \alpha_{i_{k}} \lambda_{i_{k}} \frac{\partial \varepsilon_{j i_{k}}}{\partial \lambda_{i_{k}}}(1+\mathrm{o}(1))\right. \\
& \left.-\frac{n-2}{2} \sum_{j=1}^{p} 2^{k} \alpha_{j} \alpha_{i_{k}} \frac{H_{\varepsilon}\left(a_{j}, a_{i_{k}}\right)}{\left(\lambda_{j} \lambda_{i_{k}}\right)^{\frac{n-2}{2}}}(1+\mathrm{o}(1))+R\right] .
\end{aligned}
$$

Notice that

$$
-\lambda_{i} \frac{\partial \varepsilon_{i j}}{\partial \lambda_{i}}=\frac{n-2}{2} \varepsilon_{i j}\left(1-\frac{2 \lambda_{j}}{\lambda_{i}} \varepsilon_{i j}^{\frac{2}{n-2}}\right) .
$$

Thus, if $\lambda_{i} \geqslant \lambda_{j}$ and $i, j \in T_{2}$, we have

$$
-2 \lambda_{i} \frac{\partial \varepsilon_{i j}}{\partial \lambda_{i}}-\lambda_{j} \frac{\partial \varepsilon_{i j}}{\partial \lambda_{j}} \geqslant-\lambda_{i} \frac{\partial \varepsilon_{i j}}{\partial \lambda_{i}} \geqslant \frac{n-2}{4} \varepsilon_{i j} .
$$

For $j \in T_{1}$ and $i \in T_{2}$, two cases may occur.

(i) $\frac{1}{2} d_{j} \leqslant d_{i} \leqslant 2 d_{j}$. Using in this case the fact that $j$ satisfies (3.17) and $H_{\varepsilon}\left(a_{i}, a_{k}\right) \leqslant$ $\left(d_{i} d_{k}\right)^{\frac{-(n-2)}{2}}$, we obtain

$$
\left(\frac{\lambda_{j}}{\lambda_{i}}\right)^{\frac{n-2}{2}} \varepsilon_{i j} \leqslant\left(\frac{\lambda_{j}}{\lambda_{i}}\right)^{\frac{n-2}{2}} c \sum_{k=1}^{p} \frac{H_{\varepsilon}\left(a_{k}, a_{j}\right)}{\left(\lambda_{j} \lambda_{k}\right)^{\frac{n-2}{2}}} \leqslant c \sum_{k=1}^{p}\left(\left(\lambda_{i} d_{i}\right)\left(\lambda_{k} d_{k}\right)\right)^{\frac{-(n-2)}{2}} .
$$


We deduce that

$$
\frac{\lambda_{i}}{\lambda_{j}} \varepsilon_{i j}^{\frac{2}{n-2}}=\mathrm{O}\left(\frac{1}{\left(\lambda_{1} d_{1}\right)^{2}}\right) .
$$

(ii) In other cases, we have $\left|a_{i}-a_{j}\right| \geqslant \frac{1}{2} \max \left(d_{i}, d_{j}\right)$, then

$$
\frac{\lambda_{j}}{\lambda_{i}} \varepsilon_{i j}^{\frac{2}{n-2}} \leqslant \frac{\lambda_{j}}{\lambda_{i}}\left(\lambda_{i} \lambda_{j}\left|a_{i}-a_{j}\right|^{2}\right)^{-1} \leqslant\left(\lambda_{i}\left|a_{i}-a_{j}\right|\right)^{-2}=\mathrm{O}\left(\frac{1}{\left(\lambda_{1} d_{1}\right)^{2}}\right)
$$

and (3.21) follows in this case. Using (3.18)-(3.20), and (3.21), we see that

$$
0 \geqslant((n-2) / 4) \sum_{i \in T_{2}}\left(\sum_{j \neq i} \varepsilon_{i j}-2^{p} \sum_{j=1}^{p} H_{\varepsilon}\left(a_{i}, a_{j}\right)\left(\lambda_{i} \lambda_{j}\right)^{\frac{2-n}{2}}+R\right) .
$$

Since $i \in T_{2}$ and $H_{\varepsilon}\left(a_{i} ; a_{i}\right) \sim c / d_{i}^{n-2}$ for $\varepsilon$ small enough (see [1]), then

$$
0 \geqslant c \sum_{i \in T_{2}}\left(\sum_{j \neq i} \varepsilon_{i j}+\frac{1}{\left(\lambda_{i} d_{i}\right)^{n-2}}\right)(1+\mathrm{o}(1))+R_{1} .
$$

Therefore our lemma follows.

Now, in $T_{1}$ we order all the $\lambda_{i} d_{i}: \lambda_{j_{1}} d_{j_{1}} \leqslant \lambda_{j_{2}} d_{j_{2}} \leqslant \cdots \leqslant \lambda_{j_{q}} d_{j_{q}}$. In order to simplify the notations, we suppose that $T_{1}=\{1,2, \ldots, q\}$ and $\lambda_{1} d_{1} \leqslant \lambda_{2} d_{2} \leqslant \cdots \leqslant \lambda_{q} d_{q}$.

Let us introduce the following sets:

$$
\begin{aligned}
& K_{0}=\left\{i \in T_{1} \mid \exists k_{1}, \ldots, k_{m} \in T_{1} \text { s.t. } k_{1}=i, \ldots, k_{m}=1\right. \\
& \text { and } \left.\frac{\left|a_{k_{j}}-a_{k_{j+1}}\right|}{\inf \left(d_{k_{j}}, d_{k_{j+1}}\right)} \leqslant C_{0}\right\}, \\
& B=K_{0} \cap\{1, \ldots, l\}
\end{aligned}
$$

where $l=\max \left\{i \in T_{1} \mid \lambda_{i} d_{i} / \lambda_{i-1} d_{i-1} \leqslant C_{1}\right\}$ and $C_{0}$ and $C_{1}$ are positive constants choosen later.

Lemma 3.5. - Let $B$ be defined by (3.23). Then, there exists $i \in T_{1}$ such that $i \in B$ and $i \neq 1$.

Proof. - We argue by contradiction. We assume that $B=\{1\}$. Using Proposition 3.2, and the fact that $H_{\varepsilon}\left(a_{i} ; a_{i}\right) \sim c / d_{i}^{n-2}$, we derive

$$
\begin{aligned}
0 & =\left(\nabla J\left(u_{\varepsilon}\right), \lambda_{1} \frac{\partial P \delta_{1}}{\partial \lambda_{1}}\right) \\
& =2 J(u) c_{1}\left[-\frac{(n-2)}{2} \alpha_{1} \frac{H_{\varepsilon}\left(a_{1}, a_{1}\right)}{\lambda_{1}^{n-2}}(1+\mathrm{o}(1))+\mathrm{O}\left(\sum_{k \neq 1} \varepsilon_{k 1}\right)\right] .
\end{aligned}
$$

Thus

$$
0 \leqslant-c\left(\lambda_{1} d_{1}\right)^{2-n}+\mathrm{O}\left(\sum_{k \neq 1} \varepsilon_{1 k}\right)
$$


Observe that:

- for $k \in T_{2}$, we have by Lemma 3.1, $\varepsilon_{1 k}=\mathrm{O}\left(R_{1}\right)$;

- for $k \in T_{1}$, two cases may occur.

If $k>l$, then

$$
\varepsilon_{1 k} \leqslant 2^{p+1} \sum_{j=1}^{p} \frac{H_{\varepsilon}\left(a_{k}, a_{j}\right)}{\left(\lambda_{i} \lambda_{j}\right)^{\frac{n-2}{2}}} \leqslant c\left(\frac{1}{\left(\lambda_{k} d_{k}\right)\left(\lambda_{1} d_{1}\right)}\right)^{\frac{n-2}{2}} \leqslant \frac{1}{C_{1}^{\frac{n-2}{2}}} \frac{1}{\left(\left(\lambda_{l} d_{l}\right)\left(\lambda_{1} d_{1}\right)\right)^{\frac{n-2}{2}}} .
$$

Thus $\varepsilon_{1 k}=\mathrm{o}\left(\left(\lambda_{1} d_{1}\right)^{2-n}\right)$ if we choose $C_{1}$ large enough.

In the other case, we have $k \notin K_{0}$, then $\left|a_{1}-a_{k}\right| \geqslant C_{0} \inf \left(d_{1}, d_{k}\right)$, then

$$
\varepsilon_{1 k} \leqslant\left(\frac{1}{\lambda_{1} \lambda_{k}\left|a_{1}-a_{k}\right|^{2}}\right)^{\frac{n-2}{2}} \leqslant \frac{1}{C_{0}^{n-2}} \frac{1}{\left(\left(\lambda_{1} d_{1}\right)\left(\lambda_{k} d_{k}\right)\right)^{\frac{n-2}{2}}}=\mathrm{o}\left(\frac{1}{\left(\lambda_{1} d_{1}\right)^{n-2}}\right)
$$

if we choose $C_{0}$ large enough. Thus (3.24) yields a contradiction and our lemma follows.

In order to finish the proof of the second part of Theorem 1.3, it is sufficient to prove the following lemma.

LEMMA 3.6. - For $n \geqslant 4$, we have $d^{n-2} \rho_{B} \rightarrow 0$ and $d^{n-1} \nabla \rho_{B} \rightarrow 0$, when $\varepsilon \rightarrow 0$, where $d=\inf _{i \in B} d\left(a_{i}, \partial A_{\varepsilon}\right)$ and $\rho_{B}=\rho\left(a_{i_{1}}, \ldots, a_{i_{m}}\right)$, with $B=\left\{i_{1}, \ldots, i_{m}\right\}$ the set defined by (3.23).

Before giving the proof of this lemma, we begin by studying the vector $\Lambda$ defined by

$$
\Lambda=\left(\lambda_{i_{1}}^{\frac{2-n}{2}}, \ldots, \lambda_{i_{m}}^{\frac{2-n}{2}}\right) .
$$

We denote by $e$ the eingenvector associated to $\rho_{B}$. We know that all components of $e$ are strictly positive (see [4]). Let $\eta>0$ be such that for any $\gamma$ belongs to a neighborhood $C(e, \eta)$ of $e$, we have

$$
{ }^{T} \gamma M_{B} \gamma-\rho_{B}|\gamma|^{2} \leqslant \frac{c_{2}}{d^{n-2}}|\gamma|^{2} \text { and }{ }^{T} \gamma \frac{\partial M_{B}}{\partial a_{i}} \gamma=\left(\frac{\partial \rho_{B}}{\partial a_{i}}+\mathrm{o}\left(\frac{1}{d^{n-1}}\right)\right)|\gamma|^{2}
$$

and for $\gamma \in\left(\mathbb{R}^{+}\right)^{m} \backslash C(e, \eta)$, we have

$$
{ }^{T} \gamma M_{B} \gamma-\rho_{B}|\gamma|^{2} \geqslant \frac{c_{3}|\gamma|^{2}}{d^{n-2}}
$$

where

$$
C(e, \eta) \subset\left\{y \in\left(\mathbb{R}_{+}^{*}\right)^{m} \text { s.t. }\left|\frac{y}{|y|}-e\right|<\eta\right\},
$$

$m=$ card $B_{1}$ and $M_{B}=M\left(a_{i}, i \in B\right)$ the matrix defined by (1.3). 
LEMMA 3.7. - Let $\Lambda$ be defined by (3.25). Then $\Lambda \in C(e, \eta)$.

Proof of Lemma 3.7. - We argue by contradiction. We assume that $\Lambda \in\left(\mathbb{R}_{+}^{*}\right)^{m} \backslash$ $C(e, \eta)$. Let

$$
\Lambda(t)=|\Lambda| \frac{(1-t) \Lambda+t|\Lambda| e}{|(1-t) \Lambda+t| \Lambda|e|}:=\frac{y(t)}{|y(t)|} .
$$

From Proposition 3.2, we derive

$$
\begin{aligned}
\left.\left(\nabla J\left(u_{\varepsilon}\right), Z\right)\right|_{t=0}= & -c \frac{d}{d t}\left({ }^{T} \Lambda(t) M_{B} \Lambda(t)\right)+\mathrm{O}\left(\sum_{i \in B, j \in\left(T_{1} \backslash B\right) \cup T_{2}} \varepsilon_{i j}\right) \\
& +R+\mathrm{o}\left(\frac{1}{\left(\lambda_{1} d_{1}\right)^{n-2}}\right)
\end{aligned}
$$

where $Z$ is the vector field defined on the variables $\lambda$ along the flow line defined by $\Lambda(t)$. Observe that

$$
\begin{aligned}
\left.\frac{\mathrm{d}}{\mathrm{d} t}{ }^{T} \Lambda(t) M_{B} \Lambda(t)\right)= & \frac{\mathrm{d}}{\mathrm{d} t}\left(\frac{{ }^{T} \Lambda(t) M_{B} \Lambda(t)}{|\Lambda(t)|^{2}}|\Lambda(0)|^{2}\right) \\
= & \left.|\Lambda(0)|^{2} \frac{\mathrm{d}}{\mathrm{d} t}\left(\rho_{B}+\frac{(1-t)^{2}}{|y(t)|^{2}}{ }^{T} \Lambda(0) M_{B} \Lambda(0)-\rho_{B}|\Lambda(0)|^{2}\right)\right) \\
= & |\Lambda(0)|^{2}\left(\frac{2(1-t)}{|y(t)|^{4}}\left({ }^{T} \Lambda(0) M_{B} \Lambda(0)-\rho_{B}|\Lambda(0)|^{2}\right)\right. \\
& \left.\times\left(-(1-t)|\Lambda(0)|\langle e, \Lambda(0)\rangle-t|\Lambda|^{2}\right)\right) .
\end{aligned}
$$

Thus

$$
\begin{aligned}
\left.\left(\nabla J\left(u_{\varepsilon}\right), Z\right)\right|_{t=0}= & -\frac{2 c}{|\Lambda|^{2}}\left({ }^{T} \Lambda M_{B} \Lambda-\rho_{B}|\Lambda|^{2}\right)(-|\Lambda|\langle e, \Lambda(0)\rangle) \\
& +\mathrm{o}\left(\frac{1}{\left(\lambda_{1} d_{1}\right)^{n-2}}\right)+\mathrm{O}\left(\sum_{i \in B, j \in\left(T_{1} \backslash B\right) \cup T_{2}} \varepsilon_{i j}\right) .
\end{aligned}
$$

Since $|e|=1$, then there exists k such that $e_{k} \geqslant \frac{1}{m}$. Thus

$$
\langle e, \Lambda(0)\rangle=\sum_{i} e_{i} \Lambda_{i} \geqslant \frac{1}{m} \Lambda_{k}
$$

Using (3.27), we obtain

$$
\begin{aligned}
\left.\left(\nabla J\left(u_{\varepsilon}\right), Z\right)\right|_{t=0} & \geqslant \frac{c c_{3}}{d^{n-2}}|\Lambda| \Lambda_{k}+\mathrm{o}\left(\frac{1}{\left(\lambda_{1} d_{1}\right)^{n-2}}\right)+\mathrm{O}\left(\sum_{i \in B, j \in\left(T_{1} \backslash B\right)} \varepsilon_{i j}\right) \\
& \geqslant \frac{c}{\left(\lambda_{1} d_{1} \lambda_{k} d_{k}\right)^{\frac{n-2}{2}}}+\mathrm{o}\left(\frac{1}{\left(\lambda_{1} d_{1}\right)^{n-2}}\right)+\mathrm{O}\left(\sum_{i \in B, j \in T_{1} \backslash B} \varepsilon_{i, j}\right) .
\end{aligned}
$$

Observe that 
- if $j>l$, since $j \in T_{1}$, we have

$$
\varepsilon_{i j} \leqslant \frac{c}{\left(\lambda_{1} d_{1}\right)^{\frac{n-2}{2}}} \cdot \frac{1}{\left(\lambda_{j} d_{j}\right)^{\frac{n-2}{2}}} \leqslant \frac{c}{C_{1}^{\frac{n-2}{2}}\left(\lambda_{1} d_{1} \lambda_{l} d_{l}\right)^{\frac{n-2}{2}}}=\mathrm{o}\left(\frac{1}{\left(\lambda_{1} d_{1} \lambda_{k} d_{k}\right)^{\frac{n-2}{2}}}\right) ;
$$

- if $j \notin K_{0}$

$$
\begin{aligned}
\varepsilon_{i j} & \leqslant\left(\frac{1}{\left(\lambda_{i} \lambda_{j}\left|a_{i}-a_{j}\right|^{2}\right)}\right)^{\frac{n-2}{2}} \leqslant \frac{C_{0}^{2-n}}{\left(\lambda_{1} d_{1} \lambda_{j} d_{j}\right)^{\frac{n-2}{2}}} \\
& \leqslant \frac{C_{0}^{2-n} C_{1}^{m-1}}{\left(\lambda_{1} d_{1} \lambda_{k} d_{k}\right)^{\frac{n-2}{2}}}=\mathrm{o}\left(\frac{1}{\left(\lambda_{1} d_{1} \lambda_{k} d_{k}\right)^{\frac{n-2}{2}}}\right)
\end{aligned}
$$

if we chose $C_{0} \gg C_{1}$.

Thus

$$
0 \geqslant\left(\frac{c}{\left(\lambda_{1} d_{1} \lambda_{k} d_{k}\right)^{\frac{n-2}{2}}}\right)+\mathrm{o}\left(\frac{1}{\left(\lambda_{1} d_{1}\right)^{n-2}}\right) \geqslant\left(\frac{1}{\left(\lambda_{1} d_{1}\right)^{n-2}}\right)\left(\frac{c}{\left(C_{1}\right)^{\frac{n-2}{2}}}+\mathrm{o}(1)\right)>0 .
$$

This yields a contradiction and our lemma follows.

Proof of Lemma 3.6. - Using Proposition 3.2, we have

$$
\begin{aligned}
0= & \sum_{i \in B}\left(\nabla J\left(u_{\varepsilon}\right), \lambda_{i} \frac{\partial P \delta_{i}}{\partial \lambda_{i}}\right) \\
= & \sum_{i \in B}\left[\frac{H_{\varepsilon}\left(a_{i}, a_{i}\right)}{\lambda_{i}^{n-2}}(1+\mathrm{o}(1))-\sum_{j \neq i, j \in B}\left(\varepsilon_{i j}-\frac{H_{\varepsilon}\left(a_{i}, a_{j}\right)}{\left(\lambda_{i} \lambda_{j}\right)^{\frac{n-2}{2}}}\right)(1+\mathrm{o}(1))\right. \\
& \left.+\mathrm{O}\left(\sum_{j \in\left(T_{1} \backslash B\right) \cup T_{2}} \varepsilon_{i j}\right)+R\right] \\
= & { }^{T} \Lambda M_{B} \Lambda+\mathrm{o}\left(\frac{1}{\left(\lambda_{1} d_{1}\right)^{n-2}}\right)+R_{1}+\mathrm{O}\left(\sum_{j \in\left(T_{1} \backslash B\right), i \in B} \varepsilon_{i j}\right) .
\end{aligned}
$$

Observe that, for $i \in B$ and $j \in T_{1} \backslash B$, we have, as in the proof of Lemma 3.5, $\varepsilon_{i j}=\mathrm{o}\left(\left(\lambda_{1} d_{1}\right)^{2-n}\right)$, for $C_{0}$ and $C_{1}$ large enough. Thus

$$
0={ }^{T} \Lambda M_{B} \Lambda+\mathrm{o}\left(\left(\lambda_{1} d_{1}\right)^{2-n}\right) .
$$

We assume, arguing by contradiction, that $d^{n-2} \rho_{B} \not>0$, when $\varepsilon \rightarrow 0$. Therefore, there exists $C_{4}>0$ such that $\left|\rho_{B} d^{n-2}\right| \geqslant C_{4}$.

Now, we distinguish two cases.

1st case: $\rho_{B}>0$. In this case, we derive from (3.28)

$$
0 \geqslant \rho_{B}|\Lambda|^{2}+\mathrm{o}\left(\frac{1}{\left(\lambda_{1} d_{1}\right)^{n-2}}\right) \geqslant C_{2} \frac{|\Lambda|^{2}}{d^{n-2}}+\mathrm{o}\left(\frac{1}{\left(\lambda_{1} d_{1}\right)^{n-2}}\right)>0 .
$$

This yields a conttradiction and we derive that $d^{n-2} \rho_{B} \rightarrow 0$ in this case. 
2nd case: $\rho_{B} \leqslant 0$. In this case, we derive from (3.26) and (3.28),

$$
\begin{aligned}
0 & \leqslant \rho_{B}|\Lambda|^{2}+\frac{c_{2}|\Lambda|^{2}}{d^{n-2}}+\mathrm{o}\left(\frac{1}{\left(\lambda_{1} d_{1}\right)^{n-2}}\right) \\
& \leqslant \frac{|\Lambda|^{2}}{d^{n-2}}\left(\rho_{B} d^{n-2}+C_{2}\right)+\mathrm{o}\left(\frac{1}{\left(\lambda_{1} d_{1}\right)^{n-2}}\right) \\
& \leqslant \frac{|\Lambda|^{2}}{d^{n-2}}\left(-C_{4}+C_{2}\right)+\mathrm{o}\left(\frac{1}{\left(\lambda_{1} d_{1}\right)^{n-2}}\right) .
\end{aligned}
$$

If we choose $C_{2} \leqslant \frac{1}{2} C_{4}$, we obtain a contradiction. Then $d^{n-2} \rho_{B} \rightarrow 0$, when $\varepsilon \rightarrow 0$ also in this case.

Observe that, since $d^{n-2} \rho_{B} \rightarrow 0$, then there exists $C_{5}>0$ such that $\left|a_{i}-a_{j}\right| \geqslant C_{5} d$, for any $i, j \in B$ and $i \neq j$.

We assume, arguing by contradiction, that $d^{n-1} \nabla \rho_{B} \nrightarrow \supset 0$ when $\varepsilon \rightarrow 0$.

Using Proposition 3.3, we derive

$$
\begin{aligned}
0= & { }^{T} \Lambda \frac{\partial M_{B}}{\partial a_{i}} \Lambda+\mathrm{O}\left(\sum_{j \in T_{1} \backslash B}\left(\frac{\partial \varepsilon_{i j}}{\partial a_{i}}-\frac{1}{\left(\lambda_{i} \lambda_{j}\right)^{\frac{n-2}{2}}} \frac{\partial H_{\varepsilon}}{\partial a_{i}}\left(a_{i}, a_{j}\right)\right)\right) \\
& +\mathrm{o}\left(\frac{1}{d_{i}} \frac{1}{\left(\lambda_{1} d_{1}\right)^{n-2}}\right)+\lambda_{i} R+\mathrm{O}\left(\sum_{j \in T_{1}} \lambda_{i} \lambda_{j}\left|a_{i}-a_{j}\right| \varepsilon_{i j}^{\frac{n+1}{n-2}}\right)+\mathrm{O}\left(\sum_{j \in T_{2}} \lambda_{i} \varepsilon_{i j}\right) .
\end{aligned}
$$

Observe that:

- for $j \in T_{2}$, we have by Lemma $3.1 \lambda_{i} \varepsilon_{i j}=\mathrm{O}\left(\lambda_{i} R_{1}\right)$;

- for $j \notin K_{0}$, we have

$$
\begin{aligned}
& \left|\frac{\partial \varepsilon_{i j}}{\partial a_{i}}\right|+\frac{1}{\left(\lambda_{i} \lambda_{j}\right)^{\frac{n-2}{2}}}\left|\frac{\partial H_{\varepsilon}}{\partial a_{i}}\left(a_{i}, a_{j}\right)\right| \\
& \leqslant \frac{c}{\left(\lambda_{i} \lambda_{j}\right)^{\frac{n-2}{2}}}\left(\frac{1}{\left|a_{i}-a_{j}\right|^{n-1}}+\frac{1}{d_{i}\left|a_{i}-a_{j}\right|^{n-2}}\right) \\
& \leqslant \frac{c}{C_{0}^{n-2}}\left(\frac{1}{\left(\lambda_{i} d_{i} \lambda_{j} d_{j}\right)^{\frac{n-2}{2}} d_{i}}\right)\left(\frac{1}{C_{0}}+1\right) \\
& \leqslant \frac{2 c}{C_{0}^{n-2}} \frac{1}{d} \frac{1}{\left(\lambda_{1} d_{1}\right)^{n-2}}=\mathrm{o}\left(\frac{1}{d\left(\lambda_{1} d_{1}\right)^{n-2}}\right) ;
\end{aligned}
$$

- for $j>l$, we have

$$
\begin{aligned}
& \left|\frac{\partial \varepsilon_{i j}}{\partial a_{i}}\right|+\frac{1}{\left(\lambda_{i} \lambda_{j}\right)^{\frac{n-2}{2}}}\left|\frac{\partial H_{\varepsilon}}{\partial a_{i}}\left(a_{i}, a_{j}\right)\right| \\
& \quad \leqslant \frac{c}{C_{1}^{\frac{n-2}{2}}}\left(\frac{1}{\left(\lambda_{1} d_{1}\right)^{n-2} d}\right)=\mathrm{o}\left(\frac{1}{d\left(\lambda_{1} d_{1}\right)^{n-2}}\right) ;
\end{aligned}
$$

- for $j \in T_{1}$, we have

$$
\lambda_{i} \lambda_{j}\left|a_{i}-a_{j}\right| \varepsilon_{i j}^{\frac{n+1}{n-2}}=\mathrm{o}\left(d\left(\lambda_{1} d_{1}\right)^{1-n}\right)
$$


Therefore, by (3.26) we have

$$
0={ }^{T} \Lambda \frac{\partial M_{B}}{\partial a_{i}} \Lambda+\mathrm{o}\left(\frac{1}{d\left(\lambda_{1} d_{1}\right)^{n-2}}\right)=\left(\frac{\partial \rho_{B}}{\partial a_{i}} d^{n-1}+\mathrm{o}(1)\right) \frac{|\Lambda|^{2}}{d^{n-1}}+\mathrm{o}\left(\frac{1}{d\left(\lambda_{1} d_{1}\right)^{n-2}}\right) .
$$

Thus

$$
0 \geqslant\left(\left|\frac{\partial \rho}{\partial a}\right| d^{n-1}+\mathrm{o}(1)\right) \frac{|\Lambda|^{2}}{d^{n-1}}+\mathrm{o}\left(\frac{1}{d\left(\lambda_{1} d_{1}\right)^{n-2}}\right) \geqslant C_{6} \frac{|\Lambda|^{2}}{d^{n-1}}+\mathrm{o}\left(\frac{1}{d\left(\lambda_{1} d_{1}\right)^{n-2}}\right)>0 .
$$

This yields a contradiction and our lemma follows.

\section{Proof of Theorem 1.5}

Let $\left(x_{1}, \ldots, x_{k}\right) \in A_{\varepsilon}$ such that

$$
d^{n-2} \rho_{\varepsilon}\left(x_{1}, \ldots, x_{k}\right) \rightarrow 0 \quad \text { when } \varepsilon \rightarrow 0 \text { and }\left|x_{i}-x_{j}\right| \leqslant C_{0} d, \forall i, j,
$$

where $C_{0}$ is a fixed positive constant and $d=\min _{1 \leqslant i \leqslant k} d\left(x_{i}, \partial A_{\varepsilon}\right)$.

We may assume, without loss of generality, that $d_{1}=\inf _{1 \leqslant i \leqslant k} d_{i}$.

Now we introduce the map

$$
A_{\varepsilon} \rightarrow \widetilde{A}_{\varepsilon}, \quad x \mapsto \tilde{x}=d_{1}^{-1}\left(x-x_{1}\right) .
$$

According to [1], we have

$$
\rho_{\varepsilon}\left(x_{1}, \ldots, x_{k}\right)=d_{1}^{2-n} \tilde{\rho}_{\varepsilon}\left(0, \tilde{x}_{2}, \ldots, \tilde{x}_{k}\right)
$$

where $\tilde{\rho}_{\varepsilon}$ is the function defined, replacing $A_{\varepsilon}^{k}$ by $\widetilde{A}_{\varepsilon}^{k}$ in (1.3), and $\widetilde{A_{\varepsilon}}$ converges in the $C^{1}$-topology on every compact set to $\Omega$, where $\Omega$ is a half-space or a strip.

Observe that $\left|\tilde{x}_{i}\right| \leqslant C_{0}, \forall i \in\{2, \ldots, k\}$.

Now, we have the following Lemmas.

LEMMA 4.1. - For $\varepsilon>0$, let

$$
F_{k}(\varepsilon)=\left\{\left(X_{1}, \ldots, X_{k}\right) \in \widetilde{A}_{\varepsilon}^{k} \mid \exists i \neq j \text { s.t. } X_{i}=X_{j}\right\} .
$$

Then $\tilde{\rho}_{\varepsilon}$ converges in the $C^{1}$-topology to $\rho_{\Omega}$, when $\varepsilon \rightarrow 0$, on every compact set that does not intersect $V$, where $V$ is any neighborhood of $F_{k}(\varepsilon)$ and $\rho_{\Omega}$ is the function defined, replacing $A_{\varepsilon}^{k}$ by $\Omega^{k}$ in (1.3).

The proof of Lemma 4.1 is similar to the proof of Lemma 4.1 in [1].

LemmA 4.2. - Let $\rho_{\Omega}$ the function defined replacing $A_{\varepsilon}^{k}$ by $\Omega^{k}$ in (3). Then the map

$$
] 0,1] \rightarrow \mathbb{R}, \quad t \mapsto t^{n-2} \rho_{\Omega}\left(0, t X_{2}, \ldots, t X_{k}\right)
$$

decreases when $t$ decreases for any $X_{2}, \ldots, X_{k} \in \Omega$.

The same arguments in the proof of Lemma 4.5 in [1] prove easily our lemma. From (4.1), (4.2) and Lemmas 4.1 and 4.2, we easily deduce our theorem. 


\section{Acknowledgements}

The authors would like to thank Professor Abbas Bahri, for bringing to our attention this problem, and for helpful discussions and comments.

\section{Appendix A}

In this appendix, we collect the estimates of the different integral quantities which occur in the paper. For the proof of these estimates, we refer the interested readers to [2] and [14]. In the sequel, we assume that $\varepsilon$ is small enough.

LEMMA A.1. - We have the following estimate

$$
\int_{A_{\varepsilon}}\left|\nabla P \delta_{(a, \lambda)}\right|^{2}=S_{n}-c_{1} \frac{H_{\varepsilon}(a, a)}{\lambda^{n-2}}+\mathrm{O}\left(\frac{\log \lambda d}{(\lambda d)^{n}}\right)
$$

where $c_{1}>0$ and $d=d\left(a, \partial A_{\varepsilon}\right)$. Futhermore $c_{1}$ and $\mathrm{O}$ are independent of $\varepsilon$.

LEMMA A.2. - For $n \geqslant 4$, we have the following estimate

$$
\int_{A_{\varepsilon}} P \delta^{\frac{2 n}{n-2}}=S_{n}-\frac{2 n}{n-2} c_{1} \frac{H_{\varepsilon}(a, a)}{\lambda^{n-2}}+\mathrm{O}\left(\frac{\log \lambda d}{(\lambda d)^{n}}\right)
$$

where $c_{1}$ and $\mathrm{O}$ are independent of $\varepsilon$.

LEMMA A.3. - For $i \neq j$, we have

$$
\left(P \delta_{i}, P \delta_{j}\right)_{H_{0}^{1}\left(A_{\varepsilon}\right)}=c_{1}\left(\varepsilon_{i j}-\frac{H_{\varepsilon}\left(a_{i}, a_{j}\right)}{\left(\lambda_{i} \lambda_{j}\right)^{\frac{n-2}{2}}}\right)+\mathrm{O}\left(\varepsilon_{i j}^{\frac{n}{n-2}} \log \left(\varepsilon_{i j}^{-1}\right)+\sum_{k \in(i, j)} \frac{\log \left(\lambda_{k} d_{k}\right)}{\left(\lambda_{k} d_{k}\right)^{n}}\right)
$$

where $c_{1}$ and $\mathrm{O}$ are independent of $\varepsilon$.

LEMMA A.4. - For $n \geqslant 4$, we have the following estimate

$$
\int_{A_{\varepsilon}} P \delta_{i}^{\frac{n+2}{n-2}} P \delta_{j}=\left(P \delta_{i}, P \delta_{j}\right)_{H_{0}^{1}}+R
$$

where $R=\mathrm{O}\left(\varepsilon_{i j}^{\frac{n}{n-2}} \log \left(\varepsilon_{i j}^{-1}\right)+\sum_{k \in(i, j)} \frac{\log \left(\lambda_{k} d_{k}\right)}{\left(\lambda_{k} d_{k}\right)^{n}}\right)$.

LEMMA A.5. - We have

$$
\left(P \delta, \lambda \frac{\partial P \delta}{\partial \lambda}\right)_{H_{0}^{1}}=\frac{n-2}{2} c_{1} \frac{H_{\varepsilon}(a, a)}{\lambda^{n-2}}+\mathrm{O}\left(\frac{\log (\lambda d)}{(\lambda d)^{n}}\right)
$$

where $c_{1}$ and $\mathrm{O}$ are independent of $\varepsilon$. 
LEMMA A.6. - For $n \geqslant 4$, we have

$$
\int_{A_{\varepsilon}} P \delta^{\frac{n+2}{n-2}} \lambda \frac{\partial P \delta}{\partial \lambda}=2\left(P \delta, \lambda \frac{\partial P \delta}{\partial \lambda}\right)_{H_{0}^{1}}+\mathrm{O}\left(\frac{\log (\lambda d)}{(\lambda d)^{n}}\right) .
$$

LEMMA A.7. - For $i \neq j$, we have

$$
\left(P \delta_{j}, \lambda_{i} \frac{\partial P \delta_{i}}{\partial \lambda_{i}}\right)_{H_{0}^{1}}=c_{1}\left(\lambda_{i} \frac{\partial \varepsilon_{i j}}{\partial \lambda_{i}}+\frac{n-2}{2} \frac{H_{\varepsilon}\left(a_{i}, a_{j}\right)}{\left(\lambda_{i} \lambda_{j}\right)^{\frac{n-2}{2}}}\right)+R
$$

where $R$ is defined in Lemma A.4.

LEMMA A.8. - For $n \geqslant 4$ and $i \neq j$, we have

$$
\int_{A_{\varepsilon}} P \delta_{j}^{\frac{n+2}{n-2}} \lambda_{i} \frac{\partial P \delta_{i}}{\partial \lambda_{i}}=\left(P \delta_{j}, \lambda_{i} \frac{\partial P \delta_{i}}{\partial \lambda_{i}}\right)_{H_{0}^{1}}+R
$$

where $R$ is defined in Lemma A.4.

LEMMA A.9. - For $n \geqslant 4$ and $i \neq j$, we have

$$
\frac{n+2}{n-2} \int_{A_{\varepsilon}} P \delta_{j}\left(P \delta_{i}^{\frac{4}{n-2}} \lambda_{i} \frac{\partial P \delta_{i}}{\partial \lambda_{i}}\right)=\left(P \delta_{i}, \lambda_{i} \frac{\partial P \delta_{i}}{\partial \lambda_{i}}\right)_{H_{0}^{1}}+\mathrm{O}\left(\sum_{k \in(i, j)} \frac{\log \left(\lambda_{k} d_{k}\right)}{\left(\lambda_{k} d_{k}\right)^{n}}\right) .
$$

LEMMA A.10. - We have

$$
\left(P \delta_{i}, \frac{1}{\lambda_{i}} \frac{\partial P \delta_{i}}{\partial \lambda_{i}}\right)_{H_{0}^{1}}=-\frac{1}{2} \frac{c_{1}}{\lambda_{i}^{n-1}} \frac{\partial H_{\varepsilon}}{\partial a_{i}}\left(a_{i}, a_{i}\right)+\mathrm{O}\left(\frac{1}{(\lambda d)^{n}}\right) .
$$

LEMMA A.11. - For $n \geqslant 4$, we have

$$
\int_{A_{\varepsilon}} P \delta^{\frac{n+2}{n-2}} \frac{1}{\lambda} \frac{\partial P \delta}{\partial a}=2\left(P \delta, \frac{1}{\lambda} \frac{\partial P \delta}{\partial a}\right)+\mathrm{O}\left(\frac{\log (\lambda d)}{(\lambda d)^{n}}\right) .
$$

LEMMA A.12. - For $i \neq j$, we have

$$
\begin{aligned}
\left(P \delta_{j}, \frac{1}{\lambda_{i}} \frac{\partial P \delta_{i}}{\partial a_{i}}\right)_{H_{0}^{1}}= & -\frac{c_{1}}{\left(\lambda_{i} \lambda_{j}\right)^{\frac{n-2}{2}} \lambda_{i}} \frac{\partial H_{\varepsilon}}{\partial a_{i}}\left(a_{i}, a_{j}\right)+\frac{c_{1}}{\lambda_{i}} \frac{\partial \varepsilon_{i j}}{\partial a_{i}} \\
& +\mathrm{O}\left(\sum_{k \in i, j} \frac{1}{\left(\lambda_{k} d_{k}\right)^{n}}+\varepsilon_{i j}^{\frac{n+1}{n-2}} \lambda_{j}\left|a_{i}-a_{j}\right|\right) .
\end{aligned}
$$

LEMMA A.13. - For $i \neq j$ and $n \geqslant 4$, we have

$$
\int_{A_{\varepsilon}} P \delta_{j}^{\frac{n+2}{n-2}} \frac{1}{\lambda_{i}} \frac{\partial P \delta_{i}}{\partial a_{i}}=\left(P \delta_{j}, \frac{1}{\lambda_{i}} \frac{\partial P \delta_{i}}{\partial a_{i}}\right)_{H_{0}^{1}}+\mathrm{O}\left(\sum_{k \in i, j} \frac{1}{\left(\lambda_{k} d_{k}\right)^{n}}+\varepsilon_{i j}^{\frac{n}{n-2}} \log \left(\varepsilon_{i j}^{-1}\right)\right) .
$$


LEMMA A.14. - For $n \geqslant 4$ and $i \neq j$, we have

$$
\begin{aligned}
\frac{n+2}{n-2} \int_{A_{\varepsilon}} P \delta_{j} P \delta_{i}^{\frac{4}{n-2}} \frac{1}{\lambda_{i}} \frac{\partial P \delta_{i}}{\partial a_{i}}= & \left(P \delta_{i}, \frac{1}{\lambda_{i}} \frac{\partial P \delta_{i}}{\partial a_{i}}\right)_{H_{0}^{1}}+\mathrm{O}\left(\sum_{k \in\{i, j\}} \frac{1}{\left(\lambda_{k} d_{k}\right)^{n}}\right) \\
& +\mathrm{O}\left(\varepsilon_{i j}^{\frac{n}{n-2}} \log \left(+\varepsilon_{i j}^{-1}\right)\right) .
\end{aligned}
$$

\section{REFERENCES}

[1] M. Ahmedou, K. El Mehdi, Computation of the difference of topology at infinity for Yamabe-type problems on annuli-domains, I, Duke Math. J. 94 (1998) 215-229.

[2] A. Bahri, Critical Point at Infinity in Some Variational Problems, Pitman Res. Notes Math. Ser., Vol. 182, Longman, Harlow, 1989.

[3] A. Bahri, J.M. Coron, On a nonlinear elliptic equation involving the critical Sobolev exponent: the effect of topology of the domain, Comm. Pure Appl. Math. 41 (1988) 255294.

[4] A. Bahri, Y.Y. Li, O. Rey, On a variational problem with lack of compactness: the topological effect of critical points at infinity, Calc. Var. 3 (1995) 67-93.

[5] B. Beauzamy, Introduction to Banach Spaces and Their Topology, North-Holland, 1983.

[6] H. Brezis, Points Critiques Dans les Problèmes Variationnels Sans Compacité, Séminaire Bourbaki, 40 ${ }^{e m e}$ année, Vol. 698, 1987-1988.

[7] L. Caffarelli, B. Gidas, J. Spruck, Asymptotic symmetry and local behavior of semilinear elliptic equations with critical Sobolev growth, Comm. Pure Appl. Math. 42 (1989) 271297.

[8] E.N. Dancer, A note on an equation with critical exponent, Bull. London Math. Soc. 20 (1988) 600-602.

[9] W.Y. Ding, Positive solution of $\Delta u+u^{\frac{n+2}{n-2}}=0$ on contractible domain, J. Partial Differenial Equations 2 (4) (1989) 83-88.

[10] A. Harrabi, S. Rebhi, A. Selmi, Solutions of superlinear elliptic equations and their Morse indices, I, Duke Math. J. 94 (1998) 141-157.

[11] A. Harrabi, S. Rebhi, A. Selmi, Solutions of superlinear elliptic equations and their Morse indices, II, Duke Math. J. 94 (1998) 159-179.

[12] S.S. Lin, Asymptotic behavior of positive solutions to semilinear elliptic equations on expanding annuli, J. Differential Equations 120 (2) (1995) 255-288.

[13] S. Pohozaev, Eingenfunctions of the equation $\Delta u+\lambda f u=0$, Soviet Math. Dokl. 6 (1965) $1408-1411$.

[14] O. Rey, The role of Green's function in a nonlinear elliptic equation involving critical Sobolev exponent, J. Funct. Anal. 89 (1990) 1-52.

[15] M. Struwe, Variational Methods: Applications to Nonlinear PDE \& Hamiltonian Systems, Springer-Verlag, Berlin, 1990. 